\title{
Pan-cancer stratification of solid human epithelial tumors and cancer cell lines reveals commonalities and tissue-specific features of the CpG island methylator phenotype
}

\author{
Francisco Sánchez-Vega, Valer Gotea, Gennady Margolin and Laura Elnitski
}

\begin{abstract}
Background: The term CpG island methylator phenotype (CIMP) has been used to describe widespread DNA hypermethylation at CpG-rich genomic regions affecting clinically distinct subsets of cancer patients. Even though there have been numerous studies of CIMP in individual cancer types, a uniform analysis across tissues is still lacking.

Results: We analyze genome-wide patterns of CpG island hypermethylation in 5,253 solid epithelial tumors from 15 cancer types from TCGA and 23 cancer cell lines from ENCODE. We identify differentially methylated loci that define CIMP+ and CIMP - samples, and we use unsupervised clustering to provide a robust molecular stratification of tumor methylomes for 12 cancer types and all cancer cell lines. With a minimal set of 89 discriminative loci, we demonstrate accurate pan-cancer separation of the $12 \mathrm{CIMP}+/$ - subpopulations, based on their average levels of methylation. Tumor samples in different CIMP subclasses show distinctive correlations with gene expression profiles and recurrence of somatic mutations, copy number variations, and epigenetic silencing. Enrichment analyses indicate shared canonical pathways and upstream regulators for CIMP-targeted regions across cancer types. Furthermore, genomic alterations showing consistent associations with CIMP+/- status include genes involved in DNA repair, chromatin remodeling genes, and several histone methyltransferases. Associations of CIMP status with specific clinical features, including overall survival in several cancer types, highlight the importance of the CIMP+/- designation for individual tumor evaluation and personalized medicine.
\end{abstract}

Conclusions: We present a comprehensive computational study of CIMP that reveals pan-cancer commonalities and tissue-specific differences underlying concurrent hypermethylation of $\mathrm{CpG}$ islands across tumors. Our stratification of solid tumors and cancer cell lines based on CIMP status is data-driven and agnostic to tumor type by design, which protects against known biases that have hindered classic methods previously used to define CIMP. The results that we provide can be used to refine existing molecular subtypes of cancer into more homogeneously behaving subgroups, potentially leading to more uniform responses in clinical trials.

Keywords: Cancer, DNA methylation, CpG island methylator phenotype, CIMP, TCGA, ENCODE, Pan-cancer

\footnotetext{
* Correspondence: elnitski@mail.nih.gov

Translational and Functional Genomics Branch, National Human Genome

Research Institute, National Institutes of Health, Bethesda, MD, USA
} 


\section{Background}

DNA methylation plays an important role for cell fate commitment, both in disease and normal development [1-3]. Recurrent patterns of aberrant DNA methylation are commonly observed in cancerous cells, implying that this epigenetic alteration is inherently linked to general mechanisms of oncogenesis and tumor progression [4-7]. Since methylation of specific genomic loci is a potentially actionable event, the analysis of these patterns may influence therapeutic approaches aimed at individual subtypes of tumors [8-10]. Concurrent and widespread hypermethylation of $\mathrm{CpG}$ islands in clinically distinct cancer subtypes is known as CpG island methylator phenotype (CIMP) [11,12].

The concept of CIMP was introduced more than 15 years ago within the context of colorectal cancer [13], the cancer type for which it has been most extensively studied [14-17]. Since then, CIMP occurrence has been reported in a wide variety of additional tumor types (for a review, see Hughes et al. [11]). However, evidence for a pan-cancer overlap of individual gene targets is virtually absent in these previous reports, suggesting a tissuespecific CIMP program for each type of cancer [18].

In line with this, a number of genes have been implicated in CIMP outcomes in a tissue-specific manner. For example, the inactivation of mismatch repair gene MLH1 [13] correlates strongly with CIMP in colon cancer. Glioblastoma exhibits mutations in epigenetic regulators such as $I D H 1 / 2$ and in histone encoding genes such as $H 3 F 3 A$, whereas CIMP in leukemia is associated with TET2 mutations (for a review, see Witte et al. [19]). Despite these tissue-specific differences at the level of individual genes, there is a growing body of evidence that shows increased methylation targeting certain groups of genes within some cancer types $[11,19,20]$. The methylation targets are reproducible, not random, and the actual gene subgroups are strongly associated with specific molecular and pathological features, which reinforces the targeted nature of these events. More compelling evidence points to shared similarities in pathway analyses across tumors [21,22]. For example, targets of polycomb repressor complex (PRC) are frequently identified within hypermethylated gene sets and often involve tissuespecific developmental transcription factors [23]. However, to date, no consistently methylated targets have been identified across tumor types to represent a generalizable CIMP phenotype [19] and the question of whether or not CIMP is a universal phenomenon across cancers remains unclear [11].

We present a novel approach to stratify tumors based on molecular signatures of CIMP that are evaluated in a unified manner across different cancer types. Our proposed stratification can be used to refine current molecular subtyping, with important implications in terms of translation to the clinic. We also show that methylation levels averaged across a selected set of $89 \mathrm{CpG}$ dinucleotides provide enough information to accurately distinguish CIMP+ tumors from CIMP- tumors across cancer types. This suggests that these loci are consistently targeted in CIMP across tissues and that average levels of methylation correlate to $\mathrm{CIMP}+$ status. We demonstrate numerous statistically significant associations between CIMP status, genomic functional events, and clinical annotations that recapitulate several previously known results from the literature and therefore provide a means of de facto validation that supports the adequacy of our data-driven set of CIMP labels for patient stratification. Our analysis also gives rise to new biologically plausible hypotheses to be explored in future follow-up studies.

\section{Results}

We analyzed DNA methylation data from the Illumina HumanMethylation450K platform for 5,253 solid tumors from 15 different cancer types made available by The Cancer Genome Atlas (TCGA) and for 51 cultured cell lines with known cancer $(n=23)$ or non-cancer $(n=28)$ origins made available by The Encyclopedia of Coding Elements (ENCODE). Based on reports of heterogeneous DNA methylation levels across a majority of tumor subtypes in recent studies [24-28], we hypothesize that the CIMP designation extends to subpopulations within the majority of cancer types and, therefore, that large cohorts of cancer patients may reveal a mixture of $C p G$ island methylator phenotype positive $(\mathrm{CIMP}+)$ and CpG island methylator phenotype negative (CIMP-) tumor genomes.

\section{CIMP stratification of solid tumors and human cell lines}

For each TCGA cancer type, we examined all probe locations within CpG islands (CGIs) with variable levels of DNA methylation by excluding probes with very low methylation variance $(\mathrm{SD}<0.1$, based on normalized beta values between 0 and 1). Probes located in chromosomes $\mathrm{X}$ and $\mathrm{Y}$ were also excluded from these sets. To assess the extent of aberrant hypermethylation in different types of cancer, we first analyzed genome-wide variation of CGI methylation in tumors vs. controls (Additional file 1: Figure S1 and Additional file 2). These plots visually demonstrate the distribution of methylated and unmethylated probes, whereby a large fraction of the variably methylated sites have very low levels of methylation in controls and become aberrantly hypermethylated in tumors (Additional file 1: Figure S1A). Also, a vast majority of these sites exhibit larger standard deviation in methylation levels across tumor samples than across controls (Additional file 1: Figure S1B), which is consistent with previous reports of increased methylation variability in cancer [29]. The number 
of variably methylated probes for each cancer type varied from 21,945 to 62,606 out of the 485,512 probes in the array (Table 1 ).

We examined each cancer type separately and we focused our analysis on patterns of differential methylation occurring at sites of minimal methylation in control samples and increased methylation in tumors. For this, we selected probes with average methylation levels below $5 \%$ across controls and average methylation levels above $25 \%$ across tumors. This dual-thresholding approach and the actual choice of thresholds guaranteed a very small probability of spurious detections for probe selection, as we showed using Monte Carlo simulations and random permutations of probe labels and beta-values (see the 'Methods' section). The number of differentially methylated probes selected for each cancer type ranged from 0 to 2,656 (Table 1 , the actual sets of differentially methylated probes selected for each individual cancer type are available as Additional file 3). Cases like uterine corpus endometrioid carcinoma (UCEC) and colon adenocarcinoma (COAD), with a known CIMP phenotype [13,24-26], had 1,430 and 2,656 differentially methylated probes, respectively. Thyroid carcinoma (THCA) was the only type for which no probes were selected, likely indicating no methylator phenotype within the actual samples in the data set that we used, so we excluded it from the rest of the study.

\section{Classification of tumor samples into CIMP subtypes}

We stratified samples into groups that are representative of CIMP status by classifying all tumors within each cancer type into three different categories using k-means clustering of mean methylation values computed over the tumor-specific probe sets (Figure 1A and Additional file 2). We labeled tumor samples with the lowest average levels of methylation as CIMP- and those with the highest average levels of methylation as CIMP+. We noted that clustered heat maps of the data show a gradient of DNA methylation levels across the probe sets from CIMP - to CIMP+. For the purposes of our subsequent computational and functional analysis, we focused on these two sample categories and excluded tumors assigned to the intermediate group (that we refer to as CIMPi). Eliminating samples classified as CIMPi allows unambiguous classification of tumors with strong biological differences that are most representative of CIMP extremes, at the price of a reduced effective sample size for statistical comparisons. We assessed the robustness of our sample stratification across a wide range of probe selection thresholds and found that the actual choice of cutoff values did not change our assignment of CIMP+/labels in a relevant manner (see Additional file 2). Also, the CIMP+/- groups remained largely unaltered when probes were chosen using an alternative strategy based on variance-guided feature selection (Additional file 2). The

Table 1 Cancer types, sample sizes and probe-set cardinalities

\begin{tabular}{|c|c|c|c|c|c|c|c|c|c|}
\hline Cancer type & $\begin{array}{l}\text { Variably } \\
\text { methylated } \\
\text { probes }\end{array}$ & $\begin{array}{l}\text { Differentially } \\
\text { methylated } \\
\text { probes }\end{array}$ & Control & Tumor & CIMP- & CIMPi & CIMP+ & $\begin{array}{l}\text { CIMP- } \\
\text { pan-cancer }\end{array}$ & $\begin{array}{l}\text { CIMP+ } \\
\text { pan-cancer }\end{array}$ \\
\hline BLCA (bladder urothelial carcinoma) & 49,148 & 338 & 20 & 201 & 78 & 84 & 39 & 43 & 14 \\
\hline BRCA (breast invasive carcinoma) & 46,722 & 1,311 & 96 & 676 & 270 & 244 & 162 & 76 & 47 \\
\hline COAD (colon adenocarcinoma) & 46,168 & 2,656 & 38 & 274 & 96 & 92 & 86 & 71 & 60 \\
\hline $\begin{array}{l}\text { HNSC (head and neck squamous } \\
\text { cell carcinoma) }\end{array}$ & 44,100 & 1,228 & 50 & 426 & 156 & 186 & 84 & 115 & 55 \\
\hline KIRC (kidney renal clear cell carcinoma) & 26,148 & 196 & 160 & 296 & 126 & 94 & 76 & 97 & 65 \\
\hline $\begin{array}{l}\text { KIRP (kidney renal papillary cell } \\
\text { carcinoma) }\end{array}$ & 28,083 & 40 & 45 & 147 & 60 & 59 & 28 & NA & NA \\
\hline LIHC (liver hepatocellular carcinoma) & 51,875 & 544 & 50 & 151 & 45 & 61 & 45 & NA & NA \\
\hline LUAD (lung adenocarcinoma) & 42,822 & 1,667 & 32 & 437 & 161 & 169 & 107 & 67 & 48 \\
\hline LUSC (lung squamous cell carcinoma) & 40,606 & 1,430 & 42 & 359 & 140 & 142 & 77 & 32 & 11 \\
\hline PAAD (pancreatic adenocarcinoma) & 27,899 & 1,602 & 9 & 65 & 16 & 33 & 16 & NA & NA \\
\hline PRAD (prostate adenocarcinoma) & 33,718 & 450 & 49 & 248 & 74 & 122 & 52 & NA & NA \\
\hline READ (rectum adenocarcinoma) & 40,496 & 1,255 & 7 & 96 & 31 & 39 & 26 & 22 & 22 \\
\hline STAD (stomach adenocarcinoma) & 62,606 & 1,110 & 2 & 260 & 109 & 95 & 56 & NA & NA \\
\hline THCA (thyroid carcinoma) & 21,945 & 0 & 56 & 508 & NA & NA & NA & NA & NA \\
\hline $\begin{array}{l}\text { UCEC (uterine corpus endometrioid } \\
\text { carcinoma) }\end{array}$ & 43,040 & 1,430 & 46 & 407 & 155 & 139 & 113 & 54 & 34 \\
\hline
\end{tabular}

Probe set cardinalities and sample sizes for the 15 cancer types that were included in our analysis. The last two columns show the number of CIMP+ and CIMPsamples from our genome-wide methylation study that also appear in the selected functional event data matrix from Ciriello et al. [27]. 


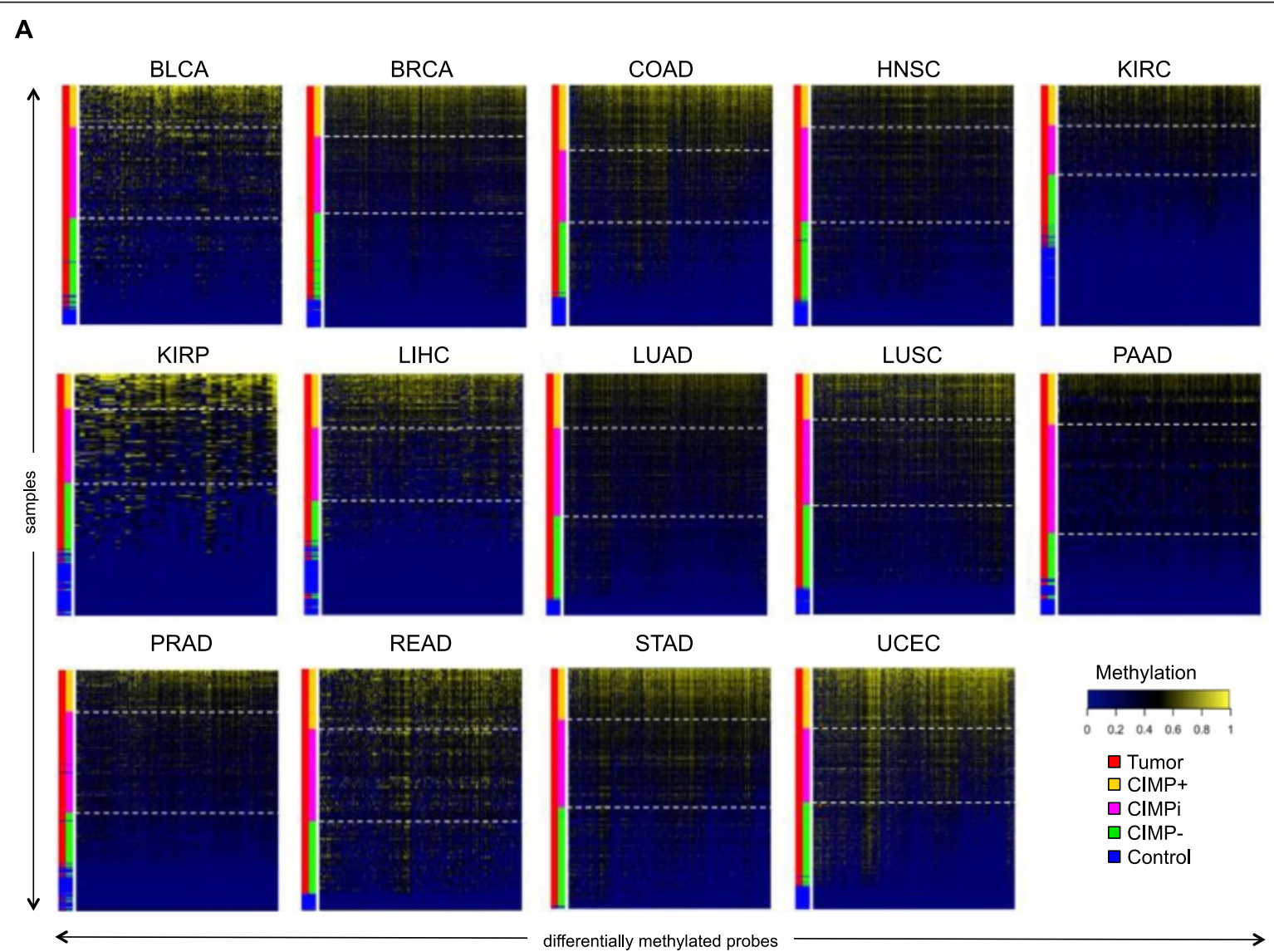

B
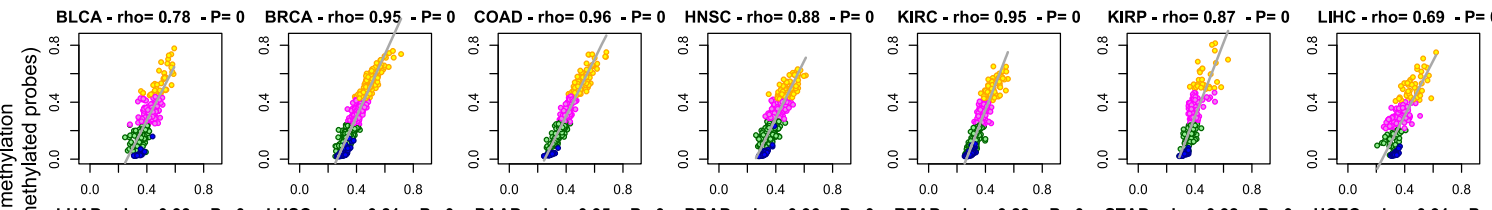

E LUAD - $r h o=0.93-P=0$ LUSC $-r h o=0.81-P=0$ PAAD $-r h o=0.95-P=0$ PRAD $-r h o=0.96-P=0 \quad R E A D-r h o=0.83-P=0$ STAD $-r h o=0.92-P=0 \quad$ UCEC $-r h o=0.91-P=0$
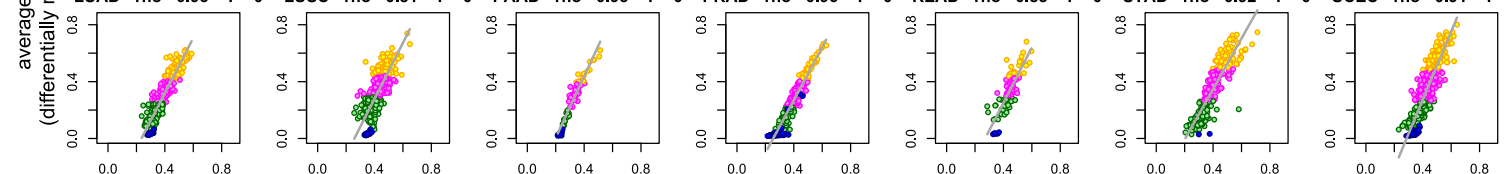

C
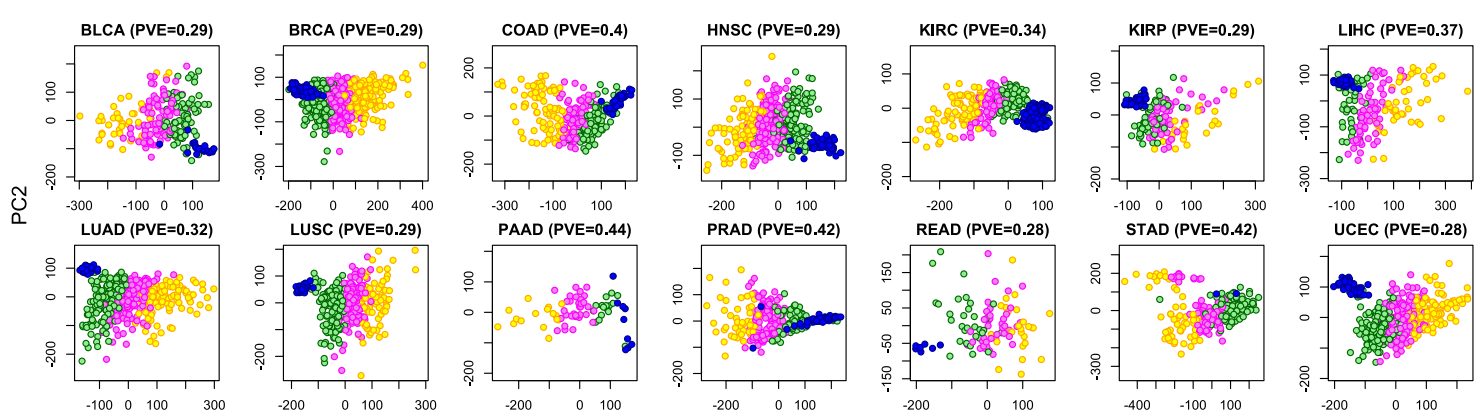

Figure 1 (See legend on next page.) 
(See figure on previous page.)

Figure $1 \mathrm{CIMP}+$ and CIMP- samples across cancer types. (A) Heat maps showing differentially methylated probes for each individual cancer type. Rows and columns represent samples and selected probes, respectively. Color side bars show tumor vs. control labels, as well as CIMP+, CIMPi, and CIMP- labels resulting from k-means clustering on the vector of average methylation values computed over differentially methylated sites. Rows were ranked from top to bottom in decreasing order of average methylation computed over selected probes. Columns were ordered horizontally using hierarchical correlational clustering. White dashed horizontal lines were used to highlight different subgroups based on CIMP status. (B) Average sample methylation computed over the sets of variably methylated probes (horizontal axes) vs. average sample methylation computed over the set of selected differentially methylated probes (vertical axes). For each plot, we provide the Spearman rho coefficient and the corresponding $P$-value. (C) PCA results where samples are projected onto the first two principal components. PCA was computed using data for all variably methylated probes within each cancer type. For each plot, we provide the corresponding percentage of variance explained (PVE) by the first two principal components. In panels (B) and (C), each point represents an individual sample and samples are colored according to their CIMP status, using the same color labels as in (A). THCA was excluded from the three panels because no differentially methylated probes had been selected for it.

number of control, tumor, CIMP+, CIMP-, and CIMPi samples for each cancer type is provided in Table 1 (individual sample labels are available as Additional file 4).

We questioned whether aberrant methylation represents a more widespread alteration of the genomic landscape by exploring correlations between the differentially methylated probe sets selected for each tissue type and the much larger sets of variably methylated probes in CpG islands (Figure 1B). These probe sets differ in size by an order of magnitude (Table 1). The strong correlation indicated that average levels of methylation measured over the relatively small sets of differentially methylated probes recapitulated the same levels measured over the larger, more extensive sets involving tens of thousands of $\mathrm{CpG}$ dinucleotides located in $\mathrm{CpG}$ islands across the genome. This conclusion indicates that a wide-spread aberrant methylation process occurs and that it can be modeled within each individual cancer type by a distinct set of differentially methylated probes that exhibit a large and consistent magnitude of effect. This finding was further supported by principal component analysis (PCA) plots computed over the large sets of variably methylated probes, where our CIMP classification labels were always grouped in spatially coherent clusters with the CIMPi tumors separating the CIMP+ from the CIMP- group (Figure 1C).

In order to further investigate similarities in CIMP across different cancer types, we drew pan-cancer heat maps of the entire set of samples, pooling together tumors and controls from different cancer and tissue types (Figure 2). We considered data from a reference set of 8,492 probes representing the union of our tissuespecific sets of differentially methylated loci. When we looked at hierarchical correlational clustering of beta values, we observed that tumors clustered according to cancer type (Figure 2A), which was consistent with previous reports of tissue-of-origin largely characterizing DNA methylation patterns in tumor cells [21]. In contrast, when we ranked samples according to their average levels of methylation over the same set of 8,492 probes, we observed that tumors clustered according to our definitions of CIMP status rather than according to cancer type (Figure 2B).

\section{Identification of a pan-cancer panel of CIMP markers}

After selecting tumor type-specific differential probe sets for individual cancer types, we searched for discriminative loci that were consistently chosen across multiple cancer types. We identified a minimal set of 89 differential probes that was present in at least 6 of 14 selected probe sets from different cancer types (Additional file 5: Table S1). This threshold of 6/14 was chosen as a tradeoff between presence in as many cancer types as possible and the need to select a sufficiently large number of probes. We performed a leave-one-type-out (LOTO) cross-validation analysis in order to assess the ability of this set of markers to separate CIMP+ from CIMPsamples. Based on Monte Carlo simulation, the classification rates and correlations with genome-wide levels of CGI methylation were statistically significant for all cancer types except kidney renal papillary cell carcinoma (KIRP) and stomach adenocarcinoma (STAD), which were consequently excluded from the rest of our study (Additional file 2). The pan-cancer panel of 89 selected loci achieved a classification accuracy of $97.57 \%$, averaged over the 12 cancer types with statistically significant classification rates (Additional file 5: Table S2).

A pan-cancer ranking of samples based on average levels of methylation computed over our proposed panel of 89 markers corroborates that this set can distinguish CIMP+ from CIMP- samples with very high accuracy (Figure 2C). Together with the pan-cancer heat maps presented earlier, these results illustrate that CIMP+ tumors show consistent elevation in average CGI methylation levels among multiple cancer types (Figure 2B,C), even though an important fraction of this hypermethylation is distributed in tissue-specific patterns (Figure 2A). Additionally, CIMP - tumors tend to have lower average methylation levels, although these are still higher than baseline non-cancer controls. The consistent behavior of the small pan-cancer panel of 89 loci across the 

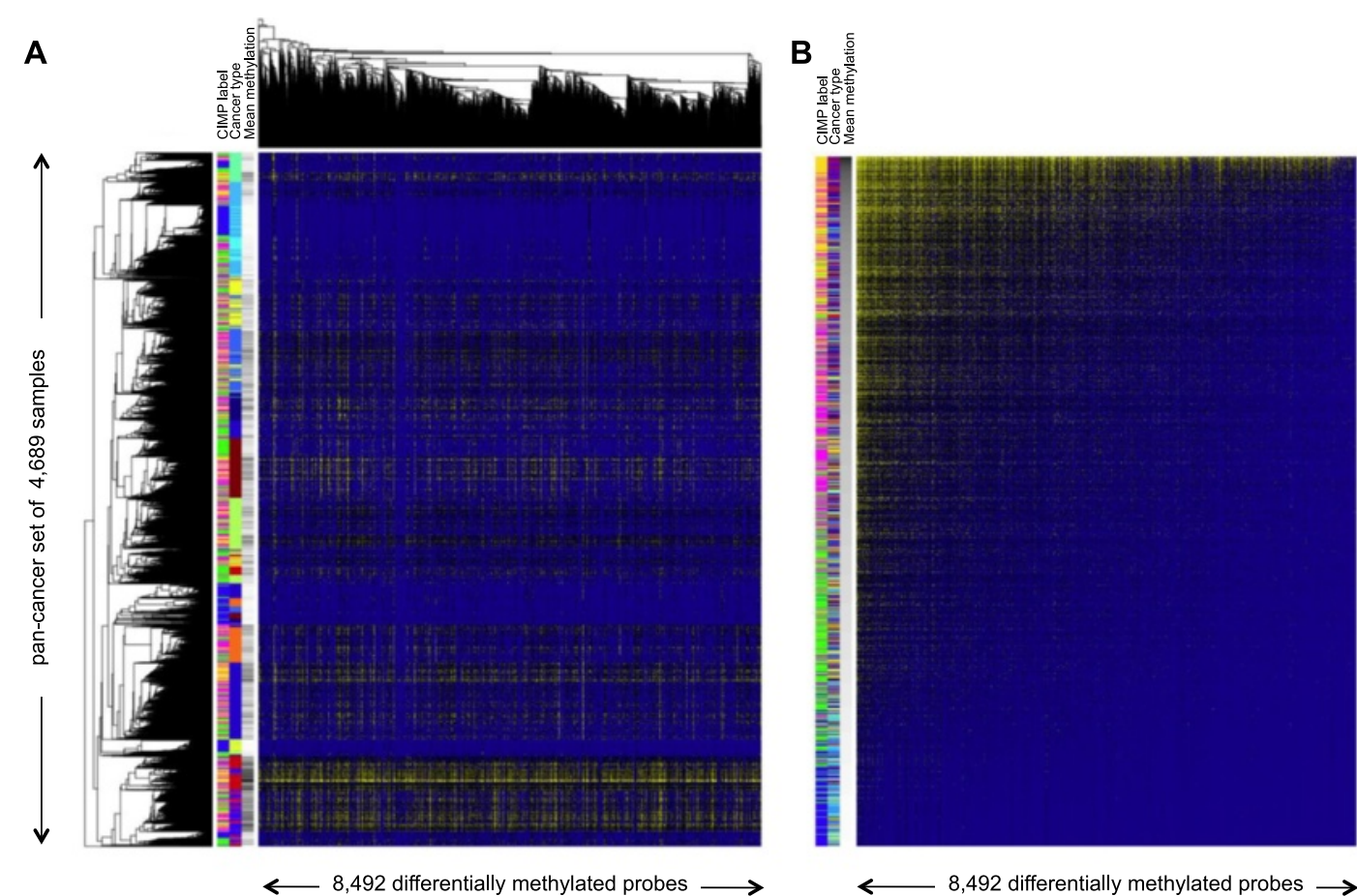

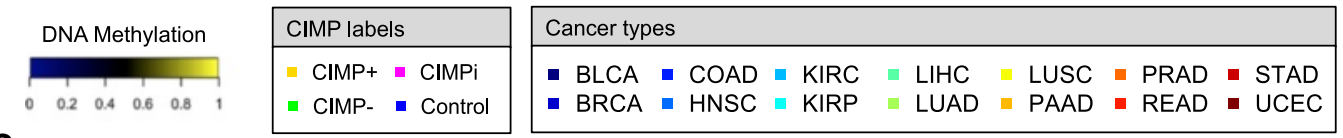

C
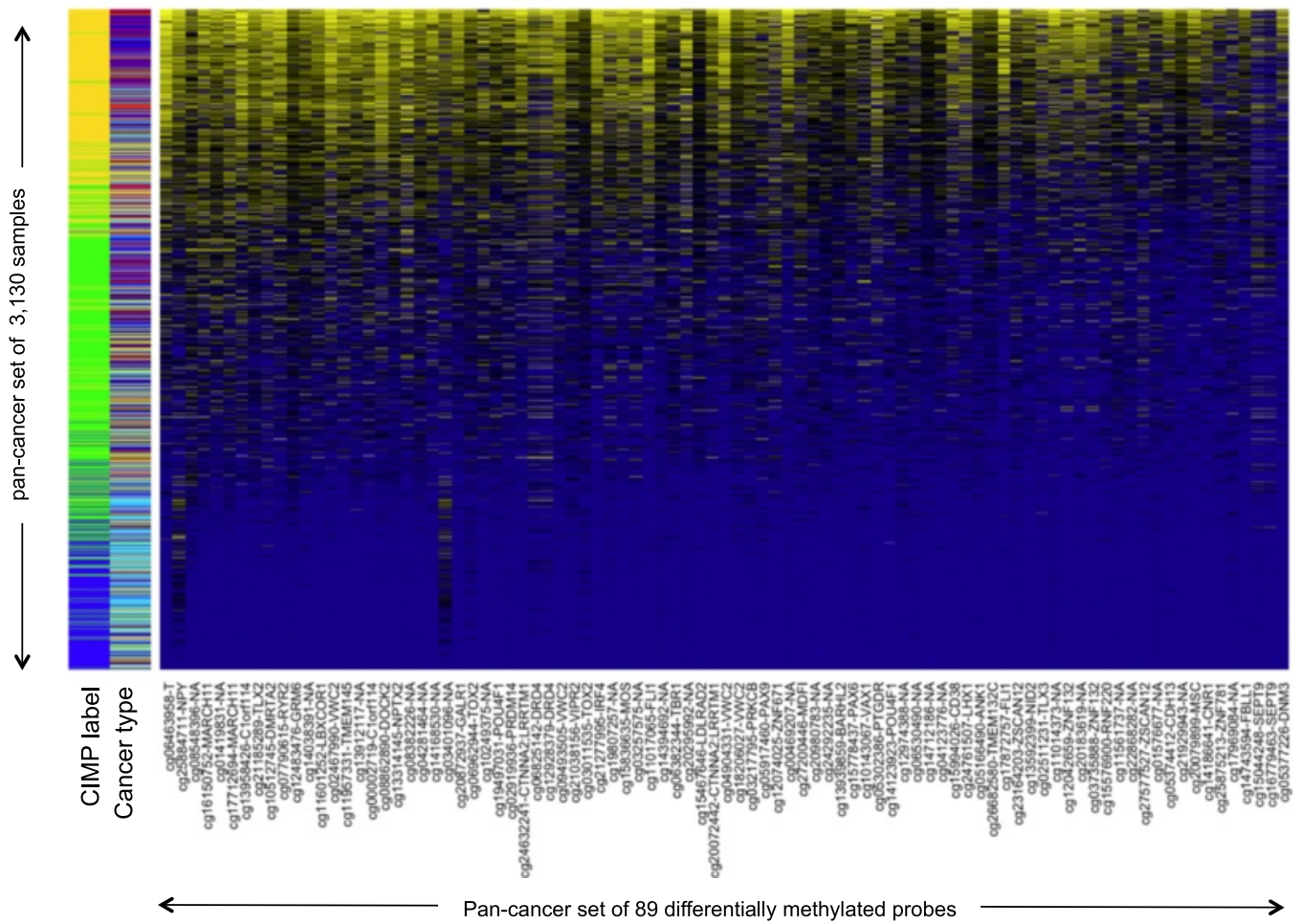

Figure 2 (See legend on next page.) 
(See figure on previous page.)

Figure 2 Pan-cancer clustering of TCGA tumors based on DNA methylation levels. Heat maps show levels of DNA methylation for TCGA tumor and control samples. Samples were pooled together across 14 cancer types (all except THCA). Each row corresponds to a sample and each column corresponds to a probe. Color bars show the CIMP status and the cancer type associated to each sample. (A) Heat map showing results for CIMP+, CIMPi, CIMP-, and control samples over a reference pan-cancer set of 8,492 probes (obtained as the union of type-specific sets of differentially methylated probes). Rows and columns were ordered using hierarchical correlational clustering. (B) Same as panel (A), but rows and columns were ranked in decreasing order of average methylation, from top to bottom and from left to right, respectively. (C) Same as panel (B), but average levels of methylation were computed using our proposed panel of 89 pan-cancer differentially methylated loci. In panels (A) and (B), a third color bar shows the relative ranking of each sample in terms of average methylation, with black showing the most methylated sample and white showing the least methylated sample. In panel (C), CIMPi tumors were excluded to facilitate visual comparison of the CIMP+/- categories and, for probes associated to known genes, the actual gene or genes are included next to each probe identifier.

evaluated cancer types demonstrates its efficacy in crosscancer determination of CIMP status.

\section{Canonical pathways and upstream regulators}

An Ingenuity Pathway Analysis (IPA) evaluation of the genes associated with the differential methylation sites in individual cancers revealed a subset of canonical pathways that are collectively targeted in the CIMP probe sets (Figure 3A). Several of these were related to tissue morphology and development, including regulation of pluripotency in embryonic stem cells. We also observed an important enrichment of genes involved in the Wnt/ beta-catenin pathway, pathways involved in glutamate receptor signaling, and regulators of epithelial-mesenchymal

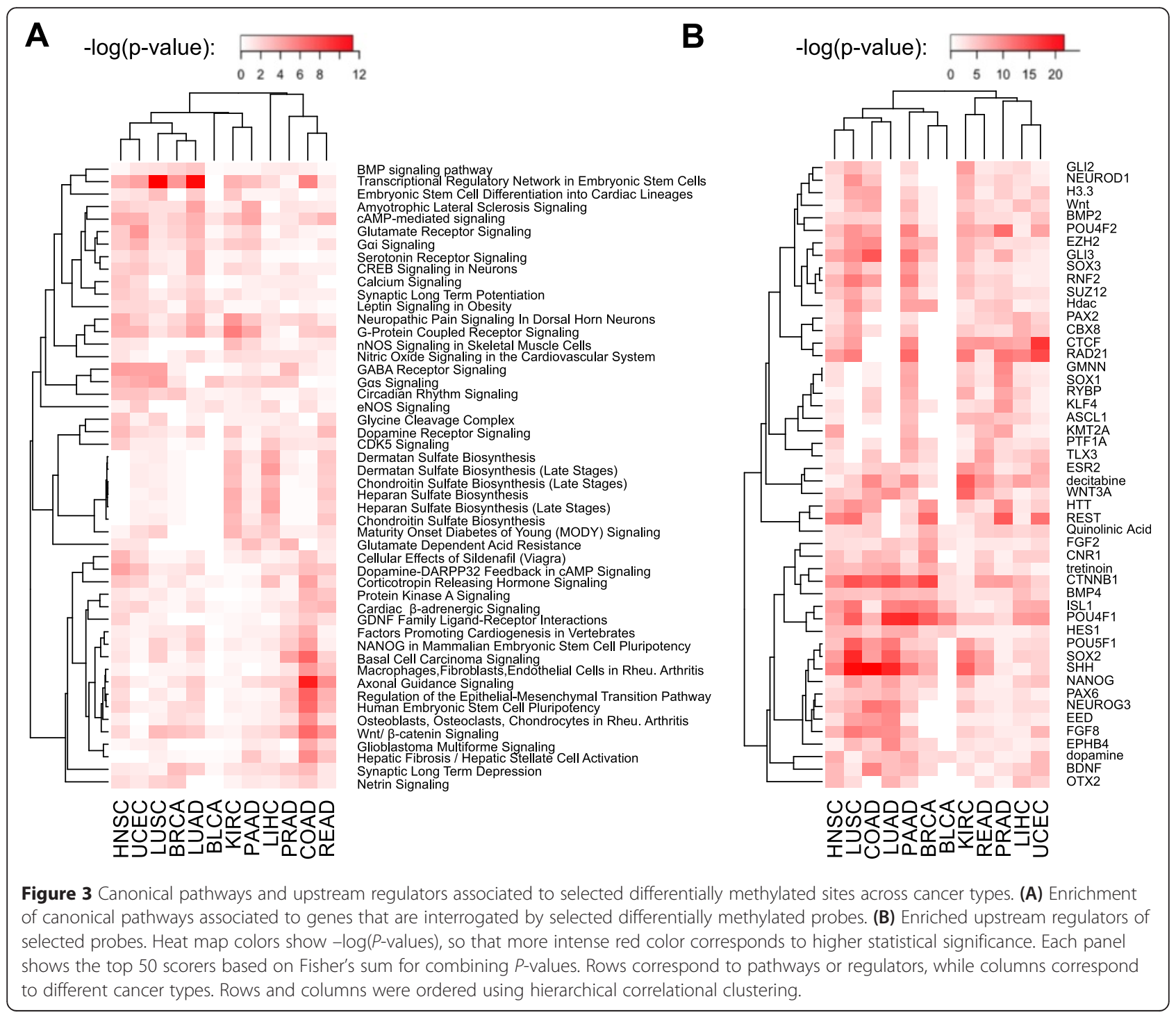



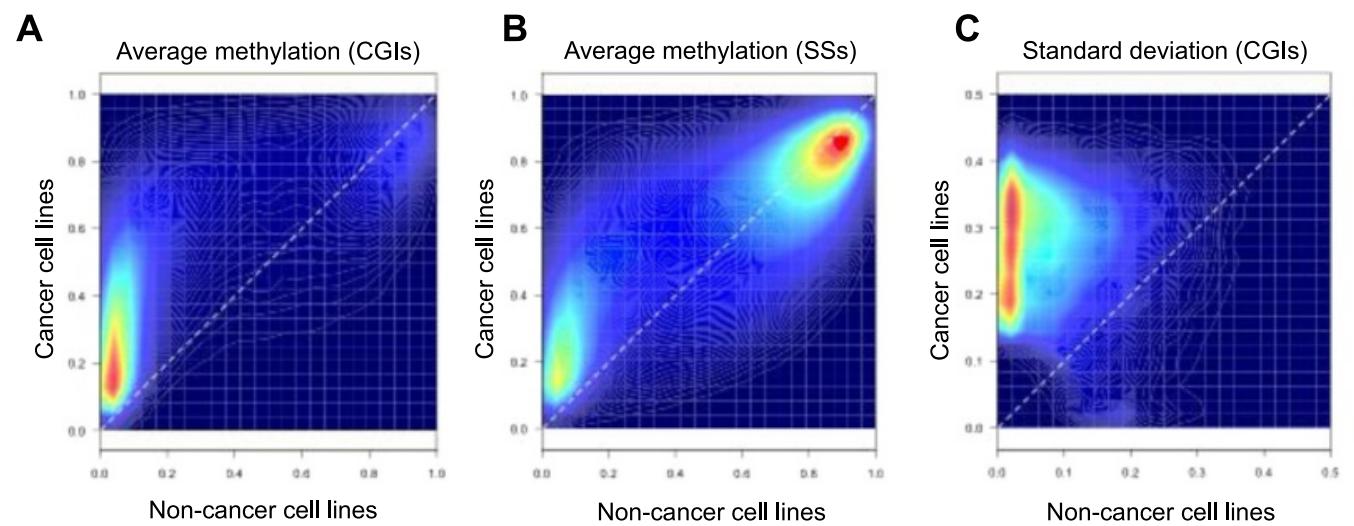

D

DNA methylation and CIMP status for ENCODE cell lines

DNA methylation

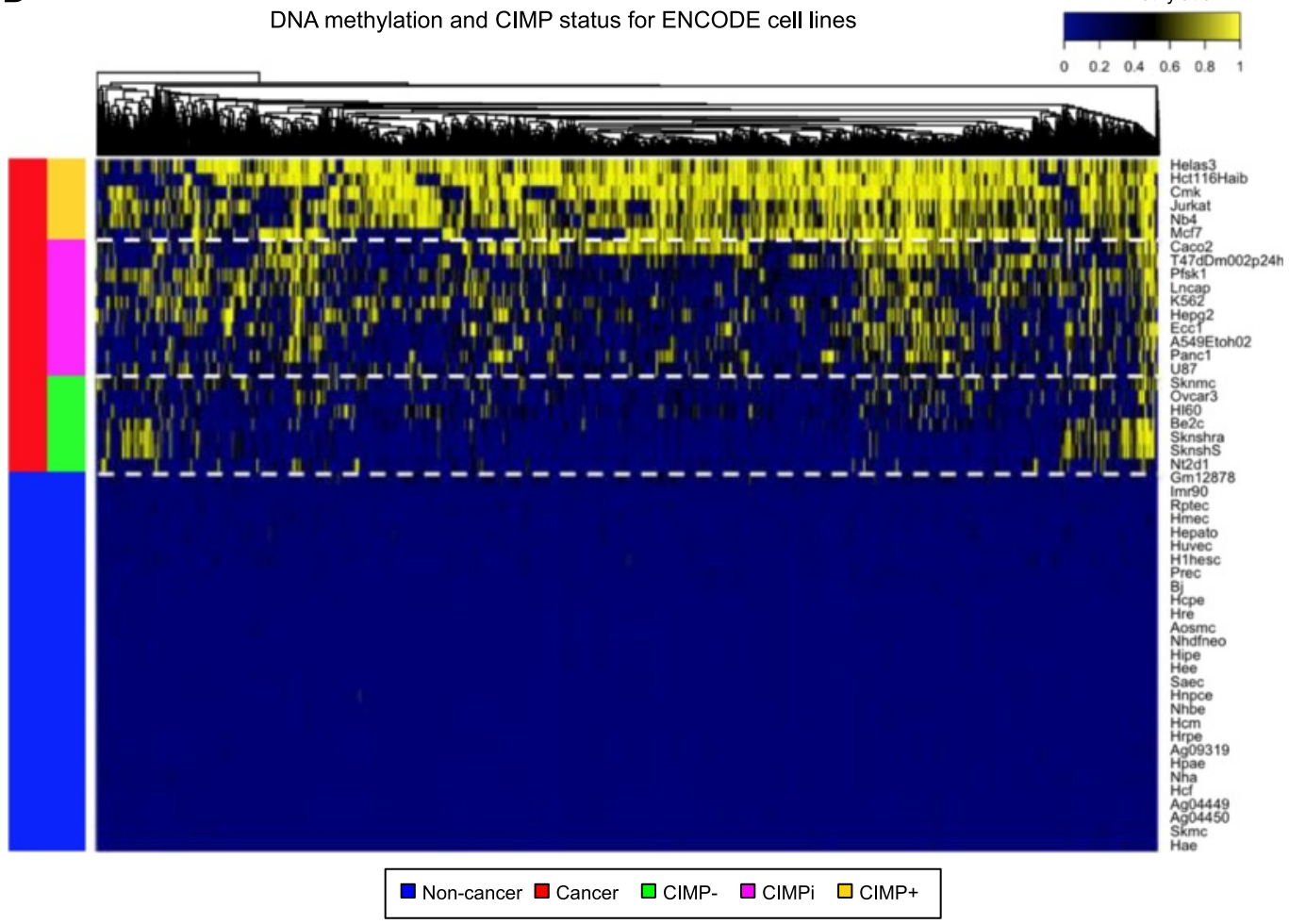

E

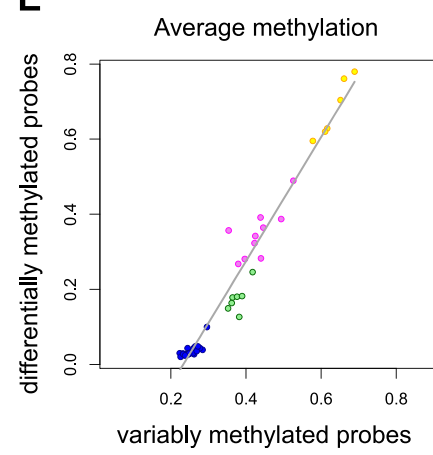

F

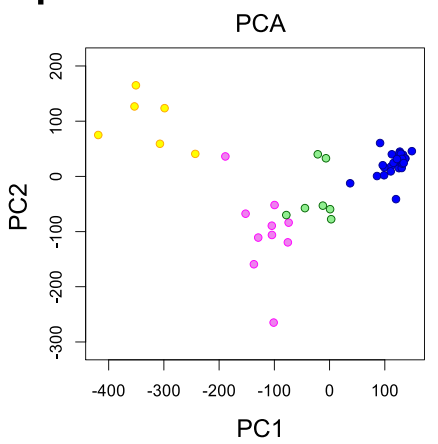

G

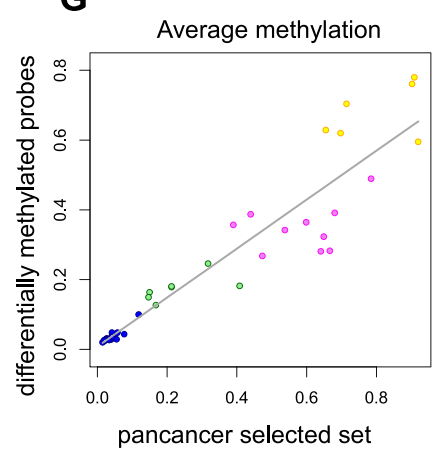

Figure 4 (See legend on next page.) 
(See figure on previous page.)

Figure 4 Characterization of CIMP in ENCODE cell lines. (A) Density plot of average site methylation for variably methylated in-CGI probes in cancer vs. non-cancer cell lines. (B) Same plot for probes in CGI shores and shelves. (C) Density plot showing standard deviation for variably methylated sites. (D) Heat map showing results from the CIMP classification algorithm. (E) Average cell line methylation computed over selected differentially methylated probes vs. average methylation computed over variably methylated probes. (F) PCA results showing samples projected onto the first two principal components and colored according to their CIMP status. (G) Average cell line methylation computed over variably methylated probes (vertical axis) vs. average methylation computed over set of 89 pan-cancer selected differentially methylated probes (horizontal axis).

transition (EMT). These pathway enrichments create potentially interesting clusters among the different tumor types, forming subgroups for head and neck squamous cell carcinoma (HNSC), UCEC, lung squamous cell carcinoma (LUSC), breast invasive carcinoma (BRCA), and lung adenocarcinoma (LUAD); kidney renal clear cell carcinoma (KIRC) and pancreatic adenocarcinoma (PAAD); and prostate adenocarcinoma (PRAD), rectum adenocarcinoma (READ), and COAD. Using the IPA tool, we also identified a set of recurrent upstream regulators for the differentially methylated probe set associated with each cancer type (Figure 3B). These included important members of the PRC (SUZ12, EZH2), chromatin remodeling genes (CTCF, HDAC), histone coding genes (H3F3A), members of the Wnt/beta-catenin pathway ( $W N T$, CTNNB1), genes known to be important for embryonic stem cell differentiation (NANOG, SOX2, POU5F1/ OCT4), and several members of the sonic hedgehog pathway (SHH, OTX2, PAX6, GLI3).

\section{Assessment of CIMP status in ENCODE cell lines}

We applied our computational pipeline for CIMP identification to methylation data from a heterogeneous panel of 51 ENCODE cell lines. We evaluated all cell lines from the ENCODE HAIB track at the UCSC Genome Browser that had a cancer or non-cancer origin $(n=23$ and $n=28$, respectively), based on available ENCODE annotations (Additional file 5: Table S3). The set of variably methylated probes included 69,186 loci. The overall methylation patterns at these sites in cancer vs. noncancer cell lines resembled our observed patterns for solid epithelial tumors compared to normal samples (Figure 4A,B,C). Our feature selection algorithm chose a set of 8,702 differentially methylated probes. K-means classification of average methylation values computed over that set identified 6, 10, and 7 cell lines as CIMP+, CIMPi, and CIMP-, respectively (Figure 4D). CIMP+ samples included HeLa and Jurkat cell lines, whereas CIMP- samples included HL60 and Nt2d1. Non-tumor cell lines were treated as controls in the feature selection algorithm, and therefore, they show baseline methylation levels at all selected probe sites. As we had observed in solid epithelial tumors, the reduced set of differentially methylated probes were highly correlated with genomewide variably methylated probes (Figure $4 \mathrm{E}$ ) and our CIMP labels revealed coherent clusters on a PCA plot
(Figure 4F). Also, we noted a strong correlation between the differentially methylated probe set and the set of 89 tumor-derived pan-cancer loci (Figure 4G), which supports the consistency of our findings between the cell lines and the tumor data. In fact, a majority of the 89 pan-cancer probes $(80 \%, 71$ of 89$)$ were included in the cell line specific, differentially methylated probe set.

\section{Pan-cancer neighborhoods of hyper- and hypomethylation}

In assessing pan-cancer patterns of aberrant methylation, we searched for consecutive probes that show consistent patterns of change. This allowed us to reduce the number of effective candidate regions that needed to be evaluated in our subsequent analysis. Using an unsupervised clustering algorithm, we identified probes with similar levels of differential DNA methylation in the CIMP+ and CIMP- samples across multiple tissues (see Additional file 2). Starting from the original set of 485,512 probes in the Illumina array, this approach identified a total of 105,875 clusters of probes that were differentially methylated at statistically significant levels for at least one of the 12 cancer types under consideration (Additional file 5: Table S4). About two thirds of the regions $(66 \%, 69,946$ of 105,875$)$ were associated with known genes (that is, fell within $1.5 \mathrm{~kb}$ of the annotated transcripts).

We divided the dataset into regions of pan-cancer hypermethylation in CIMP+ samples $($ CIMP + Hyper regions) by requiring the mean level of methylation in CIMP+ samples (averaged over all the probes within the region) to be at least 5\% higher than in CIMP- samples (that is, average differences of at least 0.05 in beta values) and that this minimum difference be observed for all 12 cancer types. We defined CIMP $+_{\text {Hypo }}$ regions in an analogous manner, but requiring that average levels of methylation in CIMP+ samples be at least 5\% lower than in CIMP- samples. The 5\% minimum difference was chosen to enforce non-negligible magnitude of effect (on top of the statistically significant differences used to define the clusters) and acted as a strong requirement when imposed simultaneously upon all the 12 cancer types. We identified 6,408 CIMP + ${ }^{\text {Hyper }}$ regions and 68 CIMP $+{ }_{\text {Hypo }}$ regions. A total of 3,892 CIMP + Hyper regions were associated with at least one gene, covering 1,805 distinct genes. A total of 54 distinct genes 


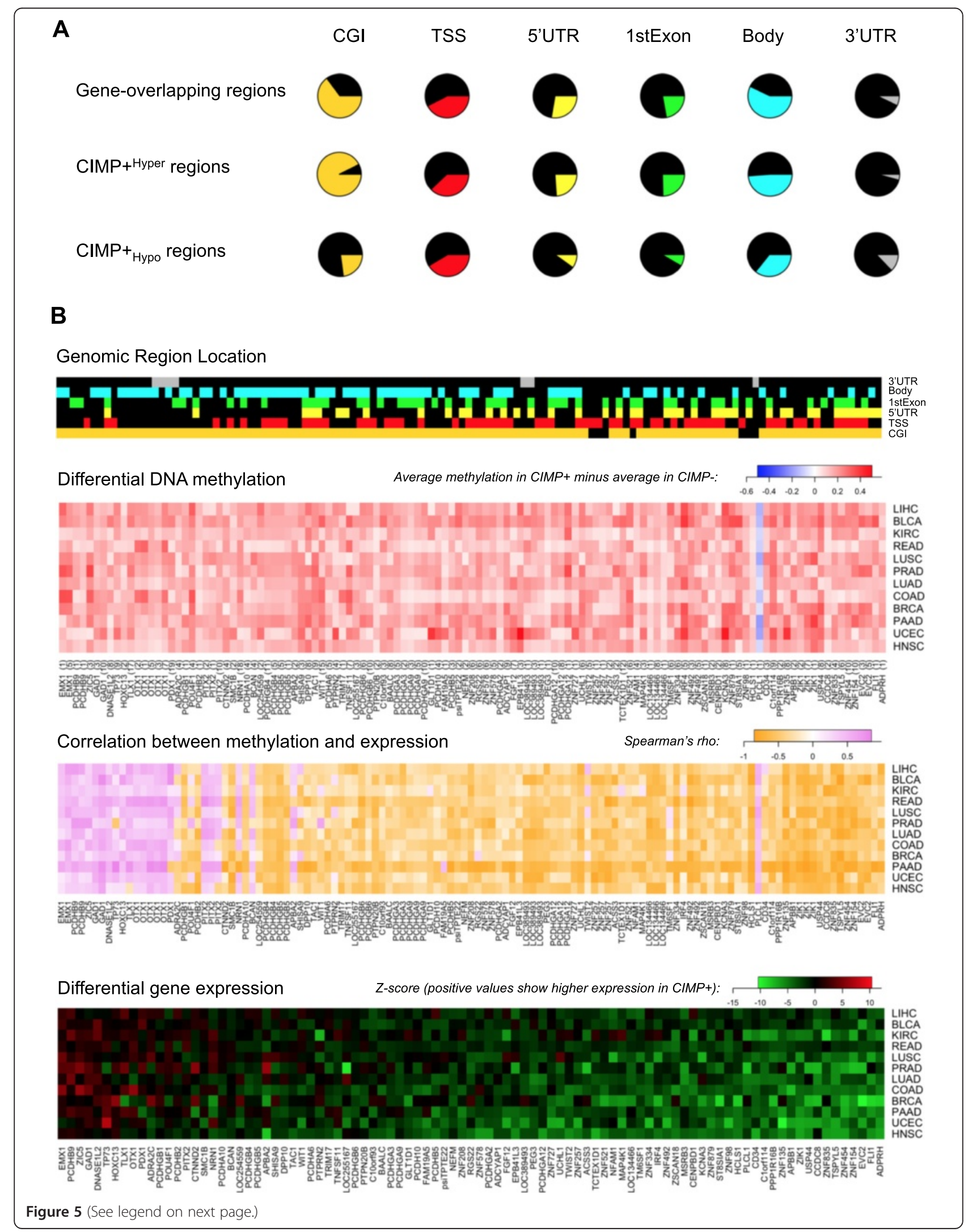


(See figure on previous page.)

Figure 5 Differentially methylated regions and differentially expressed genes in CIMP+ relative to CIMP- samples from TCGA. (A) Proportion of gene-associated regions, CIMP + ${ }^{\text {Hyper }}$ regions and CIMP + Hypo regions overlapping CGIs, TSSs, 5' UTRs, first exons, gene bodies, and 3' UTRs. (B) Differentially expressed genes exhibiting significant correlation with methylation at associated CIMP + ${ }^{\text {Hyper }}$ or CIMP + Hypo regions. The 93 genes selected in the bottom panel overlapped at least one CIMP + ${ }^{\text {Hyper }}$ or one CIMP + Hypo region and exhibited significant levels of Spearman correlation (FDR < 0.10) in all the 12 cancer types that we analyzed. Top color bars shows genomic locations of probes within each of the 120 $\mathrm{CIMP}+{ }^{\text {Hyper }}$ and $1 \mathrm{CIMP}+$ Hypo regions overlapping one of those 93 genes. Top heat map shows differences in mean methylation for these 121 regions. Middle heat map shows values of Spearman correlation between methylation within these 121 regions and expression of the 93 associated genes. Bottom panel shows differential expression (Z-scores) for these 93 genes in CIMP+ vs. CIMP- samples, with red corresponding to genes with higher expression levels in CIMP+. Rows and columns in the bottom heat map were ordered according to average Z-score, decreasing from left to right and from top to bottom. Columns in the middle and top heat map were drawn so that genes associated to differentially methylated regions were shown in the same order as in the bottom heat map. Row order was also chosen to be the same as in the bottom heat map. The number of array probes located within each CIMP + ${ }_{\text {Hyper }}$ or CIMP $+{ }_{\text {Hypo }}$ region is shown in parentheses after the corresponding gene name below the differential methylation heat map.

were associated with 54 distinct CIMP $+_{\text {Hypo }}$ regions. We identified CIMP $+{ }_{\text {Hypo }}$ and CIMP $+{ }^{\text {Hyper }}$ regions appearing concurrently within 18 genes, including five zinc-finger genes (RNF144A, ZNF727, ZNF536, ZIK1, and ZSCAN1). These discordant alterations strongly implicate disruption of the normal regulation of these genes. Consistent with our prior findings, an IPA analysis of canonical pathways and upstream regulators using these differentially methylated regions yielded results that largely coincided with the ones that we had reported using differentially methylated probes (as was shown in Figure 3). Of note, the CIMP + ${ }^{\text {Hyper }}$ region with the largest minimum increase in average methylation in every single cancer type $(\geq 30 \%)$ was associated with the Tbox transcription factor Brachyury, which has been reported to drive primary tumors towards metastasis under certain conditions by inducing EMT [30]. Of note, the probe in the pan-cancer set of 89 loci that exhibited the largest magnitude of effect in terms of average hypermethylation across samples was also associated to this gene (Figure 2C). A vast majority of CIMP + ${ }^{\text {Hyper }}$ regions overlapped CGIs, which was not the case for CIMP $+_{\text {Hypo }}$ regions (Figure 5A). Furthermore, less than half of the CIMP $+{ }^{\text {Hyper }}$ regions in the gene-associated set collocated with known transcription start sites annotated by Illumina (and a comparable number overlapped gene bodies), suggesting that aberrant hypermethylation in CIMP is not exclusive to gene promoters. However, we cannot rule out regions of unannotated alternative promoters or unannotated promoters of novel transcripts.

\section{Identification of differentially methylated and differentially expressed genes}

To test hypotheses that DNA methylation events affect gene regulatory programs, RNA-Seq data generated by TCGA were used to assess correlations between methylation and expression for genes overlapping with $C I M P+{ }_{H y p o}$ and CIMP $+{ }^{\text {Hyper }}$ regions. A subset of 121 regions associated with 93 genes exhibited significant levels of Spearman correlation between methylation and expression in all 12 cancer types, with varying magnitudes of effect in terms of actual differential expression (Figure 5B). The gene with the strongest global difference in gene expression was $A D P R H$, which is involved in DNA repair through histone ADP-ribosylation [31] and has been shown to play a role in tumorigenesis in mice [32]. Additionally, FLI1 (which had been identified in our selected differentially methylated probe sets for 8 of 14 cancer types), contained a combination of CIMP + ${ }^{\text {Hyper }}$ and $C I M P+{ }_{\text {Hypo }}$ regions, which occurred at the gene promoter and the first exon, respectively. Only one CIMP $+_{\text {Hypo }}$ region exhibited consistent levels of significant correlation with expression across all 12 cancer types, located at the 3'UTR of gene PLCL1. We also identified families of genes showing consistently significant correlations between expression and differential methylation, including 14 genes from the protocadherin family and 16 zinc-finger genes. In particular, ZNF154 - which we recently proposed as a pan-cancer biomarker to distinguish tumors from non-cancer controls [28] - was also part of this collection.

\section{Analysis of associations between methylator phenotype and composite functional profiles}

One hypothesis to explain the occurrence of CIMP across different tissues is that it arises due to some shared underlying genomic alterations or functional mechanisms. To address commonalities, we used characterizations of TCGA data generated by Ciriello et al. [27], which consisted of 479 selected functional events (SFEs) including 116 copy number gains, 151 copy number losses, mutation of 199 genes, and epigenetic silencing of 13 genes (requiring promoter methylation and decreased expression). Although not specifically reported as driver alterations, these events had originally been chosen due to their relevance in cancer. The study intersected 9 of the 15 TCGA tumor collections from our analysis: bladder urothelial carcinoma (BLCA), BRCA, COAD, HNSC, KIRC, LUAD, LUSC, READ, and UCEC.

For each cancer type, we compared the average frequencies associated with each functional event in CIMP+ 
samples and CIMP- samples. From a matrix of differential frequencies, we selected the top 100 functional events with the highest average absolute differences (Figure 6A). Events with strong effects in more than one tumor type included MGMT and MLH1 promoter methylation, as well as mutation of ARID1A, KRAS, BRAF, and PTEN. Events that were strong but gave mixed results towards the CIMP phenotype included mutation of TP53, PIK3CA, $F B X W 7$, and several amplification and deletion regions. We performed an aggregation analysis where we looked

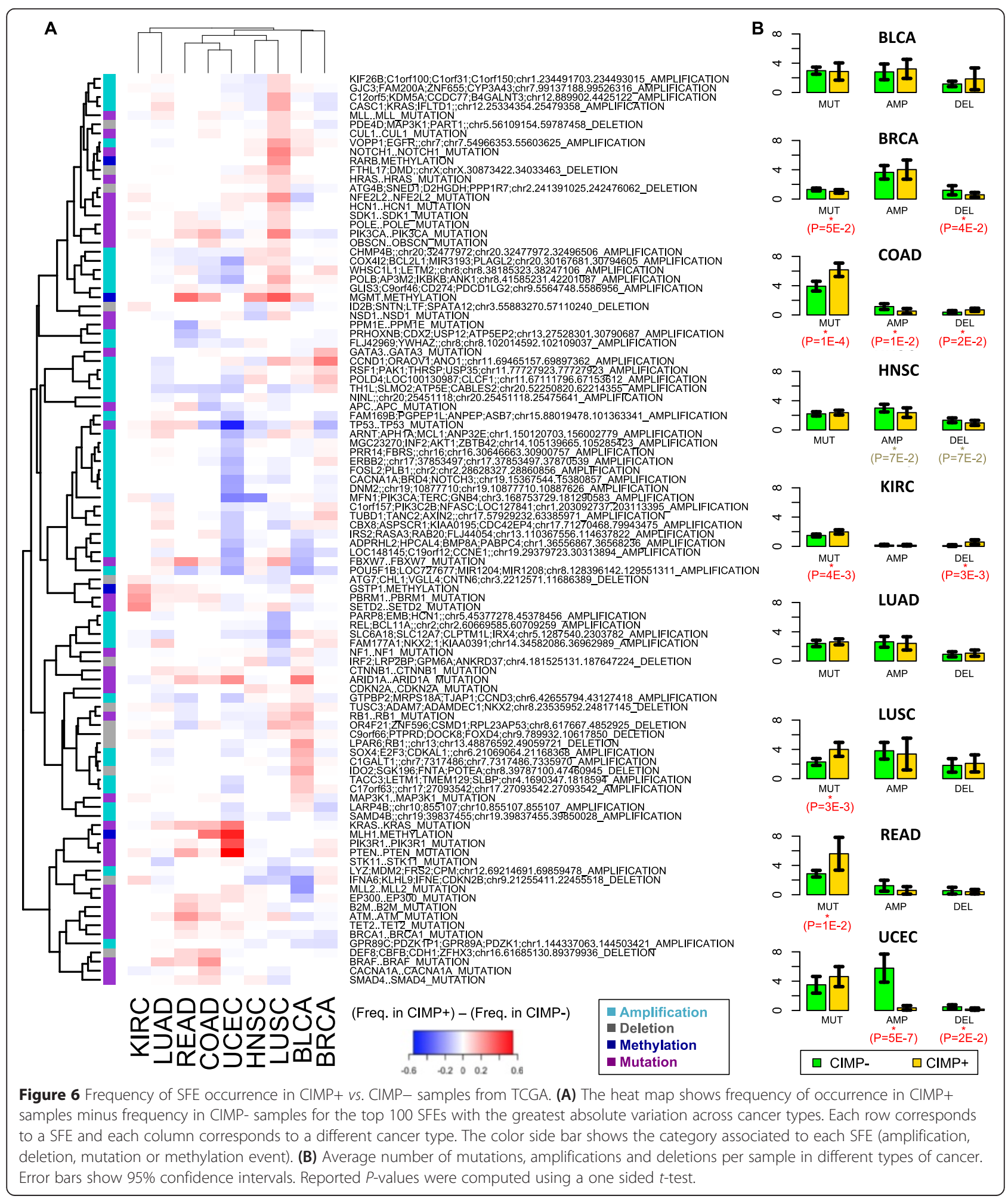


for differences in SFE frequencies between the entire set of pooled CIMP+ samples and the entire set of pooled CIMP- samples (including $356 \mathrm{CIMP}+$ samples and 577 CIMP- samples from all 9 cancer types combined) to test for the possibility of universal or convergent pathway events. The top 20 SFEs in terms of differences in frequencies are shown in Table 2, wherein a total of 12 SFEs showed statistically significant differences (FDR $<0.10)$. Of these, four amplification events were significantly more frequent in CIMP- samples and involved genes such as PIK3CA, TERC, SOX2, CCNE1, BRD4, and NOTCH3. In contrast, mutations in six genes (BRAF, PTEN, KRAS, SETD2, PIK3R1, and PBRM1) and two gene silencing events (MLH1 and MGMT, for which the smallest FDRs were recorded) were more frequent in CIMP+ samples. Of interest within the context of CIMP, PBRM1 is a SWI/ SNF chromatin remodeling complex gene that has been reported to play a tumor suppressor role across multiple cancer types [33], and SETD2 is a histone methyltransferase for H3K36 methylation, which is enriched in the gene bodies of actively transcribed genes [34]. The remaining mutations are well known due to their involvement in the PI3K/PTEN/AKT/mTOR [35] and the Ras/Raf/MEK/ERK [36] pathways. In particular, both BRAF [37] and KRAS $[38,39]$ mutations have been linked to CIMP status (high and low, respectively) in colorectal cancer [37-39]. Despite these associations, the mechanistic connections to CIMP are not discernable from the reported functional activities of these proteins and the prominent driver mutations do not appear to be responsible for a 'universal' methylator phenotype.

We compared the average counts of copy number events and mutational events per sample in the CIMP+ and the CIMP- subsets for each individual cancer type (Figure 6B). CIMP+ samples for COAD, KIRC, LUSC, and READ exhibited a larger number of mutational events per sample than CIMP- samples, implicating impairment of DNA repair processes. In contrast, copy number variation showed significant effects in CIMPsamples, where amplifications occurred more frequently in COAD and UCEC tumors, and deletions occurred more frequently in BRCA and UCEC tumors. However,

Table 2 Top 20 SFEs in terms of differential frequencies in pooled CIMP+ vs. pooled CIMP- samples

\begin{tabular}{|c|c|c|c|c|c|c|c|}
\hline Selected functional event & DifFreq & Count CIMP+ & Freq CIMP+ & Count CIMP- & Freq CIMP- & $P$-value & FDR \\
\hline MLH1.METHYLATION & 0.100 & 38 & 0.107 & 4 & 0.01 & $7.11 \times 10^{-13}$ & $3.40 \times 10^{-10}$ \\
\hline MGMT.METHYLATION & 0.113 & 58 & 0.163 & 29 & 0.05 & $2.22 \times 10^{-08}$ & $5.30 \times 10^{-06}$ \\
\hline $\begin{array}{l}\text { MFN1.PIK3CA.TERC.GNB4.SOX2.MECOM. } \\
\text { ZMAT3.KCNMB3.ZNF639.KCNMB2..chr3. } \\
\text { 168753729.181290583_AMP }\end{array}$ & -0.094 & 17 & 0.048 & 82 & 0.14 & $3.06 \times 10^{-06}$ & $4.89 \times 10^{-04}$ \\
\hline BRAF..BRAF_MUTATION & 0.063 & 30 & 0.084 & 12 & 0.02 & $1.15 \times 10^{-05}$ & $1.38 \times 10^{-03}$ \\
\hline PTEN..PTEN_MUTATION & 0.080 & 47 & 0.132 & 30 & 0.05 & $2.48 \times 10^{-05}$ & $2.38 \times 10^{-03}$ \\
\hline KRAS..KRAS_MUTATION & 0.091 & 62 & 0.174 & 48 & 0.08 & $4.07 \times 10^{-05}$ & $3.25 \times 10^{-03}$ \\
\hline SETD2..SETD2_MUTATION & 0.053 & 25 & 0.070 & 10 & 0.02 & $5.86 \times 10^{-05}$ & $4.01 \times 10^{-03}$ \\
\hline $\begin{array}{l}\text { LOC148145.C19orf12.CCNE1..chr19. } \\
\text { 29379723.30313894_AMP }\end{array}$ & -0.044 & 3 & 0.008 & 30 & 0.05 & $2.02 \times 10^{-04}$ & $1.21 \times 10^{-02}$ \\
\hline $\begin{array}{l}\text { CACNA1A.BRD4.NOTCH3.. } \\
\text { chr19.15367544.15380857_AMP }\end{array}$ & -0.035 & 1 & 0.003 & 22 & 0.04 & $2.98 \times 10^{-04}$ & $1.59 \times 10^{-02}$ \\
\hline PIK3R1..PIK3R1_MUTATION & 0.046 & 25 & 0.070 & 14 & 0.02 & $1.10 \times 10^{-03}$ & $5.28 \times 10^{-02}$ \\
\hline DNM2..chr19.10877710.10887626_AMP & -0.024 & 0 & 0.000 & 14 & 0.02 & $1.46 \times 10^{-03}$ & $6.35 \times 10^{-02}$ \\
\hline PBRM1..PBRM1_MUTATION & 0.058 & 43 & 0.121 & 36 & 0.06 & $2.37 \times 10^{-03}$ & $9.46 \times 10^{-02}$ \\
\hline ATM..ATM_MUTATION & 0.042 & 25 & 0.070 & 16 & 0.03 & $2.82 \times 10^{-03}$ & $1.01 \times 10^{-01}$ \\
\hline TP53..TP53_MUTATION & -0.101 & 143 & 0.402 & 290 & 0.50 & $2.94 \times 10^{-03}$ & $1.01 \times 10^{-01}$ \\
\hline ARID1A.ARID1A_MUTATION & 0.052 & 40 & 0.112 & 35 & 0.06 & $6.16 \times 10^{-03}$ & $1.95 \times 10^{-01}$ \\
\hline GSTP1.METHYLATION & 0.054 & 44 & 0.124 & 40 & 0.07 & $6.51 \times 10^{-03}$ & $1.95 \times 10^{-01}$ \\
\hline SAMD4B..chr19.39837455.39850028_AMP & -0.024 & 2 & 0.006 & 17 & 0.03 & $1.47 \times 10^{-02}$ & $3.76 \times 10^{-01}$ \\
\hline $\begin{array}{l}\text { TMED11P.CTBP1.SPON2.. } \\
\text { chr4.1105313.1243877_DEL }\end{array}$ & -0.016 & 0 & 0.000 & 9 & 0.02 & $1.55 \times 10^{-02}$ & $3.76 \times 10^{-01}$ \\
\hline KEAP1..KEAP1_MUTATION & -0.016 & 0 & 0.000 & 9 & 0.02 & $1.55 \times 10^{-02}$ & $3.76 \times 10^{-01}$ \\
\hline FOSL2.PLB1..chr2.28628327.28860856_AMP & -0.017 & 0 & 0.000 & 10 & 0.02 & $1.65 \times 10^{-02}$ & $3.76 \times 10^{-01}$ \\
\hline
\end{tabular}


these events are not always positively correlated, as shown by the reduction in deletions in COAD CIMPsamples.

Finally, we used binary classification and regression trees on these SFEs in order to identify individual features that were able to recursively partition the original set of tumors into increasingly homogeneous subgroups based on either CIMP status (in the classification case, Figure 7) or average methylation levels from the variably methylated probe sets (in the regression case, Additional file 1: Figure S2). We learned pan-cancer trees by pooling together samples across different cancer types (Figure 7 and Additional file 1: Figure S2), and we also learned type-specific trees on individual cancer types (Additional file 1: Figures S3 and S4). The pan-cancer trees highlight
SFEs which are relevant in more than one type of tumor. For example, $M L H 1$ promoter methylation is observed in a subset of COAD and UCEC tumors with a very strong majority of CIMP+ labels. Similarly, a high proportion of CIMP+ labels was observed in samples with $M G M T$ promoter methylation, combined with either (a) $F B X W 7$ mutations or (b) APC and KRAS mutations or (c) absence of $F B X W 7$ and $A P C$ mutations (Figure 7). Of note, subgroups containing these alterations consisted entirely of tumors of the aero-digestive tract (HNSC, LUSC, COAD, and READ). Notably, MLH1 and MGMT have been previously associated with two distinct methylation landscapes in colorectal cancer that exhibited important differences in terms of KRAS and APC mutation frequency [40].

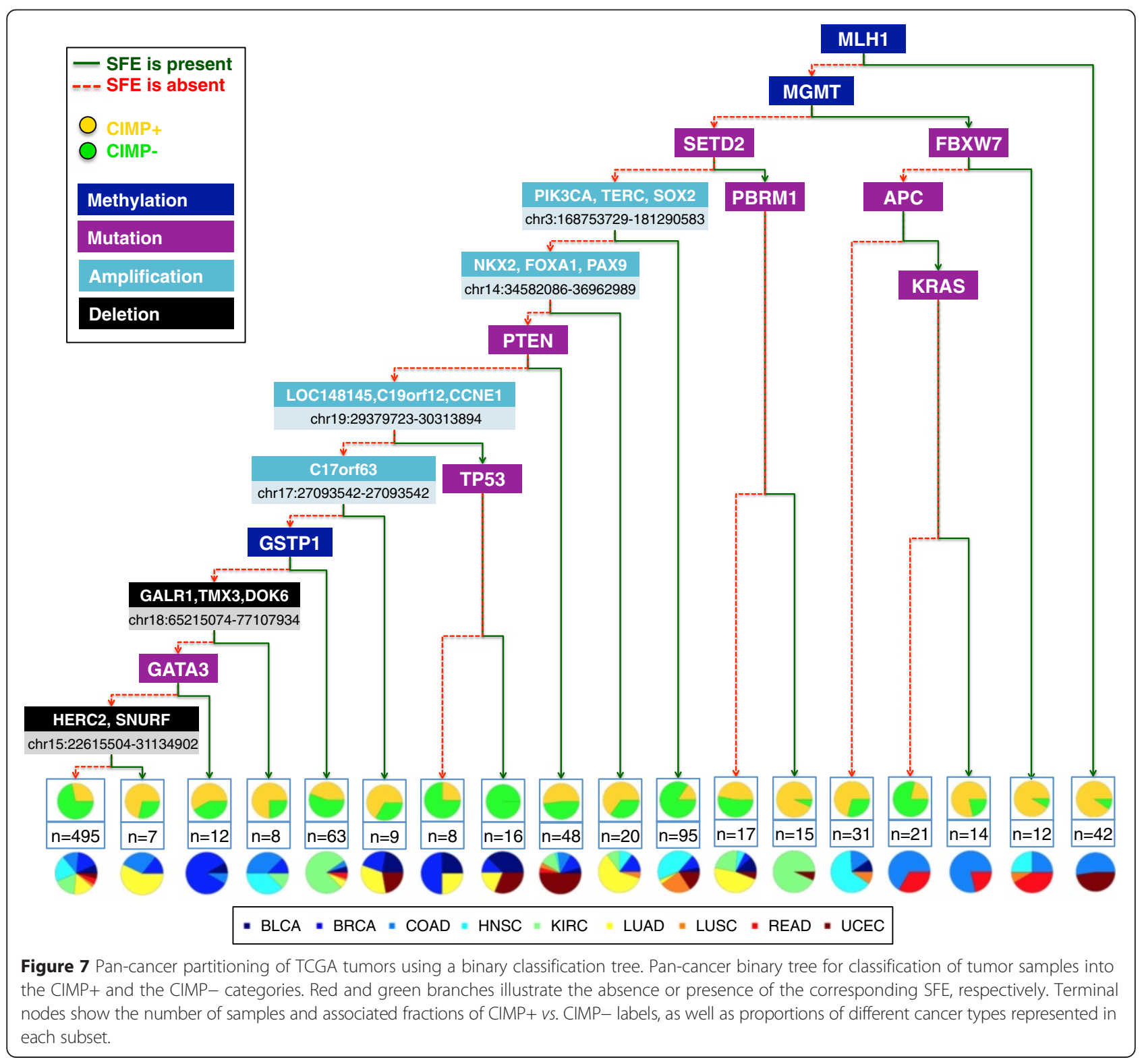


In our samples lacking $M G M T$ and $M L H 1$ promoter methylation, the highest proportions of CIMP+ samples were observed in a subgroup dominated by KIRC tumors that were characterized by a combination of SETD2 and PBRM1 mutations. We found co-occurrence of CCNE1 amplification and TP53 mutations in a subgroup derived from a mixture of BLCA, BRCA, UCEC, and LUAD tumors where all the samples were labeled as CIMP-. By contrast, within these same cancer types, amplification of a chromosomal region around C17orf63 in tumors lacking amplification of CCNE1 was observed in a subgroup that contained a higher proportion of CIMP+ than CIMP- labels. Other copy number events, such as amplification of the region containing NKX2 and FOXA1 or deletion of HERC2 were observed in subgroups with a majority of CIMP+ samples and a large fraction of LUAD but also a few BRCA tumors. Deletion of a region containing GALR1 was observed in a subset with a majority of CIMP+ tumors that came primarily from the COAD and HNSC types.

Our pan-cancer regression tree shows that VHL mutations correlate with significant reductions in average levels of CGI methylation in KIRC tumors (Additional file 1: Figure S2). Similarly, amplification of two chromosomal regions in chromosome 17, including ERBB2 (a.k.a. $H E R 2$ ), co-occurs with an overall increase in CGI methylation in a subgroup consisting mostly of BRCA samples with some LUSC representation. Mutations observed in the context of decreased average methylation are NSD1 in HNSC and KDM6A (sharing a mixed subgroup with a majority of BLCA tumors).

Individual tumor trees were also associated with recurrent functional events. For example, our classification tree for BLCA highlights alterations affecting $R B 1$ and ARID1A in CIMP+ tumors (Additional file 1: Figure S3), consistent with previous independent analyses [19,41]. In BRCA, we found a strong association between CCND1 amplification and CIMP status (Additional file 1: Figures S3 and S4). Also, the presence of $M Y C$ amplifications delineated a subset of samples that consisted entirely of CIMP- tumors (Additional file 1: Figure S3). This is consistent with reports from TCGA identifying $M Y C$ amplification and high-expression in basal-like breast tumors, which tend to be hypomethylated [42]. In KIRC, the presence of either mutations or deletions affecting gene SETD2 and methylation of the GSTP1 promoter correlate with an important increase in the frequency of CIMP+ cases (Additional file 1: Figures S3 and S4). Also in KIRC, we found that deletion of a genomic region containing $C D K N 2 A$ and $C D K N 2 B$ on chromosome 9 is associated with increased levels of CGI methylation (Additional file 1: Figure S4). This kind of deletion has been linked to a more aggressive phenotype of clear cell carcinoma [43]. In LUSC, methylation of the
$R B P 1$ promoter and amplification of a region containing KDM5A correlate with an increase in average CGI methylation (Additional file 1: Figure S4). In UCEC, our data show that methylation of the $M L H 1$ promoter results in a very high probability of CIMP+ status. For samples that do not exhibit this trait, the presence of TP53 mutations is associated with the opposite outcome. Among the remaining samples, PIK3R1 mutations are linked to increased CIMP+ rates (Additional file 1: Figure S3). Thus, the presence of tumor-specific mutations provides a potential link to predicting methylation status.

\section{Analysis of associations between methylator phenotypes and clinical features}

We compared our sets of data-driven CIMP labels with clinical annotations provided by TCGA for individual samples. First, we note that the sets of controls used in our analysis covered a range of ages that is similar to the range of ages covered by the sets of tumor samples for most cancer types (Figure 8A). This fact rules out age effects as a confounding factor because our feature selection algorithm requires selected differentially methylated probe sites to remain consistently low across controls, leading to large overlaps in the ages associated with the three CIMP categories (Figure 8A). In fact, ANOVA results using the Kruskal-Wallis test fail to reject the null hypothesis of equal median ages for different CIMP subgroups in 9 out of 12 types (after applying Holm's correction for multiple hypotheses). The only exceptions are BRCA, COAD, and KIRC. For these three types, the median age of CIMP+ patients is higher than the median age in CIMP- (consistent with an independent study of CIMP+ status in COAD [11]). We found no statistical association between CIMP status and gender in any cancer type, except KIRC ( $P=0.025$, Fisher's exact test with Holm's correction), where we observed a significantly higher frequency of CIMP+ labels in male samples (45\%, 58 of 128$)$ than in female samples $(22 \%, 15$ of 66$)$.

Whereas mutational events are correlative, an association of survival and CIMP status would indicate relevant subclasses of tumors that could prompt more narrowly defined intervention strategies. We evaluated survival curves based on CIMP status for the 12 cancer types in our study and found significant differences in KIRC, LUSC, and UCEC (BRCA results were borderline significant at 0.06 ) (Figure $8 \mathrm{~B})$. CIMP+ samples exhibited better survival curves than CIMP- samples in UCEC and LUSC, whereas CIMP- samples exhibited better survival than CIMP+ in KIRC and BRCA. Of course, these results must be interpreted carefully because they result from a univariate analysis that does not explicitly take into account potentially confounding factors.

Consistent with prior knowledge, we also detected a significant level of statistical association between CIMP 


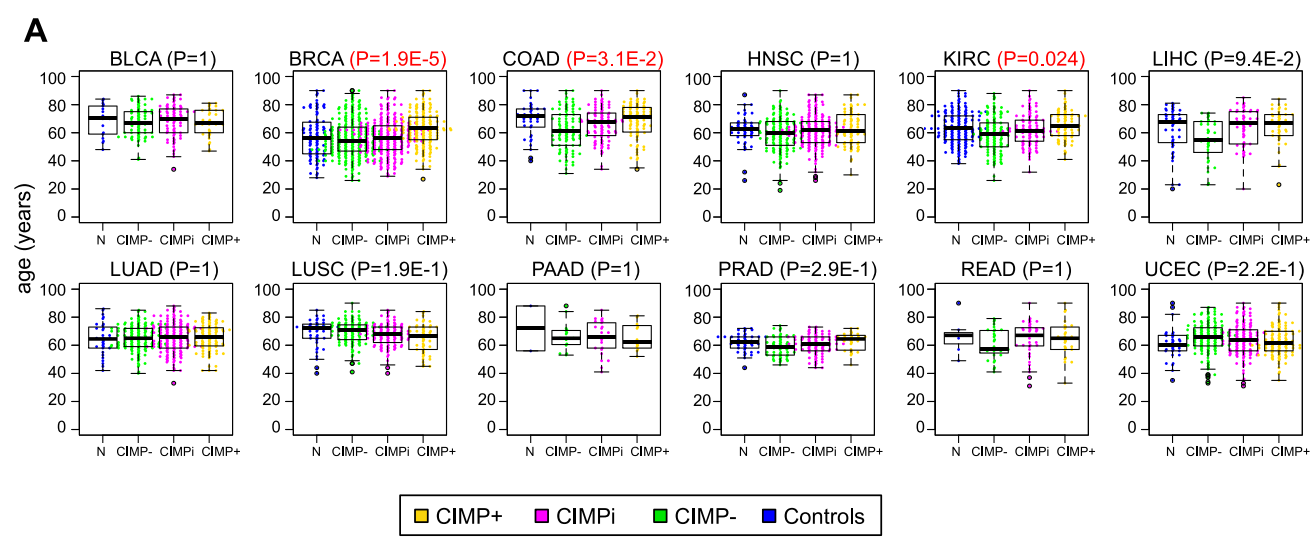

B
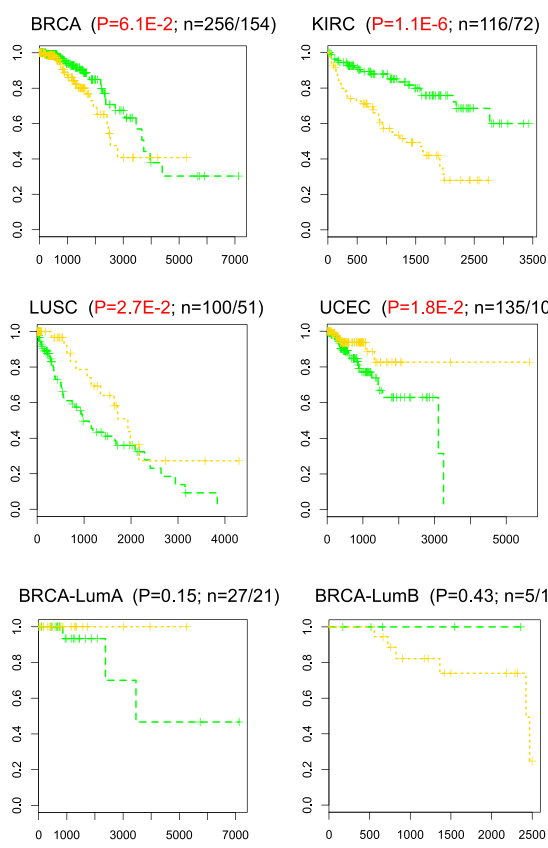

time (days)
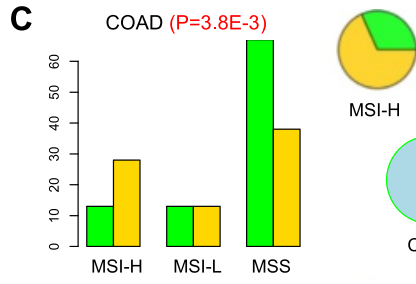

MSI-H
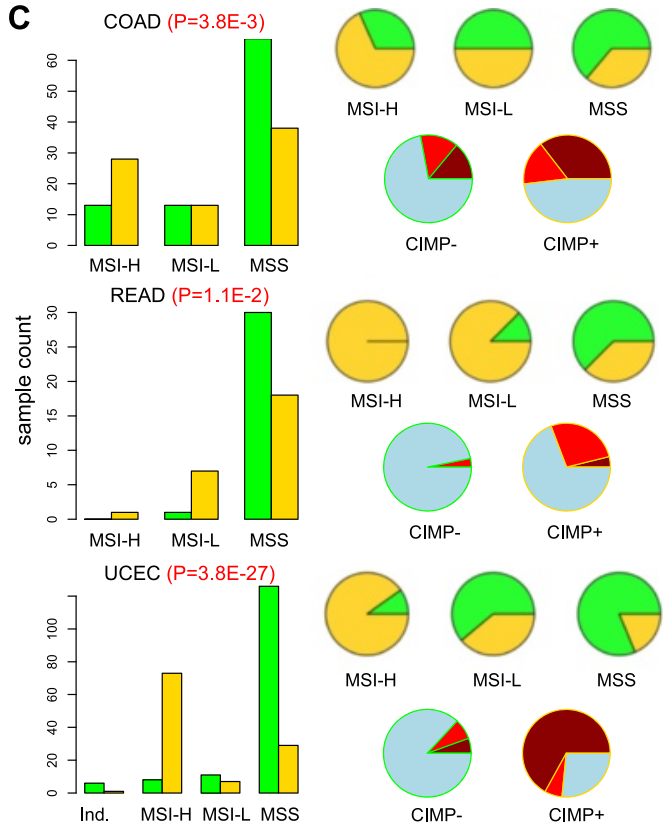

MSI-H
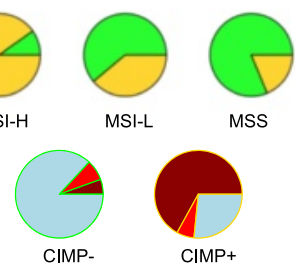

$\square$ CIMP+ $\mathrm{CIMP}-\square \mathrm{MSI}-\mathrm{H} \quad \square \mathrm{MSI}-\mathrm{L} \quad \square \mathrm{MSS}$
D

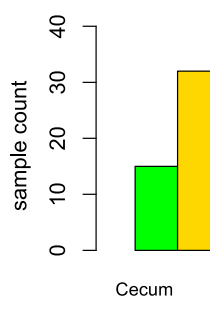

들

(1)

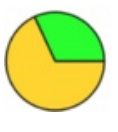

COAD - Anatomic subdivision ( $P=2.4 \mathrm{E}-6)$
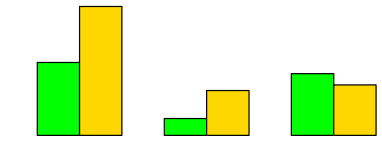
Ascending
Colon

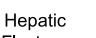

$\begin{array}{cc}\text { Hepatic } & \text { Transverse } \\ \text { Flexture } & \text { Colon }\end{array}$

(3) (4)
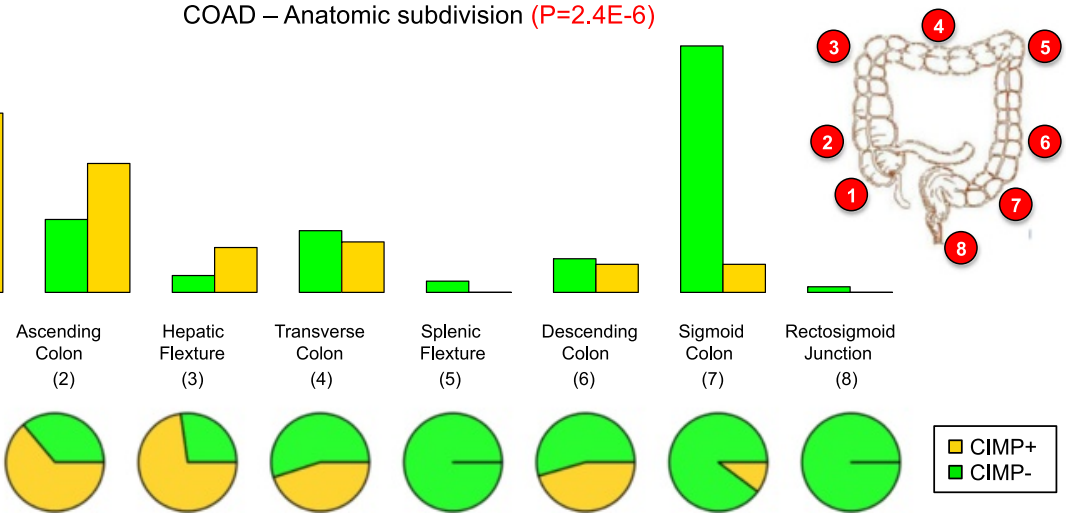

Rectosigmoid
Junction

(8)

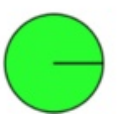

$\square$ CIMP+ $\square$ CIMP-

Figure 8 (See legend on next page.) 
(See figure on previous page.)

Figure 8 Associations between CIMP status and clinical annotations. (A) Age vs. CIMP status across 12 cancer types. (B) Overall survival curves for the four cancer types exhibiting significant differences based on CIMP status (BRCA, KIRC, LUSC, UCEC) and overall survival curves for luminal A and luminal B subtypes in BRCA based on CIMP status. (C) Microsatellite instability vs. CIMP status in COAD, READ, and UCEC. (D) CIMP status as a function of anatomic subdivision in COAD. P-values come from a Kruskal-Wallis test for difference in medians in panel (A), a log-rank test for survival curve differences in panel (B), and Fisher's exact test in panels (C) and (D). For each survival curve in (B), the number of CIMP-/CIMP+ samples is provided next to the corresponding $P$-value.

status and microsatellite instability (MSI) in the three cancer types for which this annotation was available (Figure $8 \mathrm{C})$. These included COAD $\left(P=3.8 \times 10^{-3}\right.$; Fisher's test with Holm's correction), READ $(P=0.011)$, and UCEC $\left(P=3.8 \times 10^{-27}\right)$. In all cases, a vast majority of CIMP- samples were microsatellite stable while CIMP+ labels were more frequent among microsatellite instable samples. These results are consistent with independent reports of MSI in CIMP-high colorectal tumors [37] and also with the division between UCEC serous and endometrioid samples (largely CIMP- and CIMP+ in our analysis, respectively), where endometrioid tumors carry microsatellite instability and serous tumors do not [26]. Thus, the MSI characteristic appears to be associated with CIMP+ status and mutually exclusive with TP53 mutations and copy number variation.

We observed statistically significant levels of association between our CIMP labels and the five categories commonly used to catalogue breast tumors [42] (Additional file 1: Figure S5A; $P=3 \times 10^{-8}$, Fisher's exact test). In effect, the basal and normal-like categories were enriched in CIMP- samples, the luminal B category consisted mostly of CIMP+ and the luminal A group contained a more balanced mixture of CIMP types. This conclusion is consistent with trends reported by TCGA, where many luminal B samples showed a hypermethylator phenotype while basal-like samples were hypomethylated and associated with very high rates of TP53 mutations [42]. Still, treatment approaches for breast cancer often consider hormone responsiveness over subtype classification. In BRCA samples (Additional file 1: Figure S5A), CIMP+ appeared more frequently in ER+ samples than in ERsamples $\left(P=6.7 \times 10^{-5}\right.$, Fisher's exact test, Bonferroni correction). Likewise, a large number of HER2+ tumors were CIMP+, suggesting applications for methylation inhibitors in combination therapy of CIMP+ tumors. In contrast, a majority of HER2- samples were CIMP- $(P=0.001$, Fisher's exact test, Bonferroni correction).

In COAD (Figure 8D), we observed a strong association between CIMP status and anatomic neoplasm subdivision $\left(P=2.4 \times 10^{-6}\right.$, Fisher's exact test, Bonferroni correction). More precisely, there is a progressive decrease in the frequency of CIMP+ samples along the intestinal tract from cecum, through ascending, transversal, and descending colon and finishing at the rectosigmoid junction. This variation appears to be proportional to distance along the intestinal tract and is consistent with a previous study of colorectal cancer samples collected from three anatomic locations and assessed at eight CIMP-specific promoters using MethyLight technology [44], as well as independent reports of a gradual decrease in the frequency of $B R A F$ mutations and microsatellite instability within this same region of the intestinal tract [45].

In HNSC (Additional file 1: Figure S5B), we also observed a significant level of association between CIMP labels and anatomic subdivision $\left(P=5.0 \times 10^{-3}\right.$, Fisher's exact test, Bonferroni correction), with more CIMP+ than CIMP- samples at the oral cavity, buccal mucosa, and floor of the mouth. However, CIMP- labels outnumbered CIMP+ labels in the base of tongue, alveolar ridge, tonsil, and larynx. Also in HNSC, CIMP- samples exhibited significantly better survival curves for recurrence free status than CIMP+ samples (Additional file 1: Figure S5B).

In KIRC (Additional file 1: Figure S6A), samples labeled as CIMP+ tended to have higher grade $\left(P=2.5 \times 10^{-6}\right.$, Fisher's exact test, Bonferroni correction) and higher pathological stage $\left(P=1.5 \times 10^{-12}\right.$, Fisher's exact test, Bonferroni correction). They also exhibited higher $\mathrm{T}$ parameters $\left(P=8.4 \times 10^{-12}\right.$, Fisher's exact test, Bonferroni correction) and $\mathrm{M}$ parameters $\left(P=5.6 \times 10^{-4}\right.$, Fisher's exact test, Bonferroni correction) based on the TMN cancer staging notation system, which implies larger primary tumor sizes and higher distant metastatic spread, respectively. Together with the survival curves shown in Figure $8 \mathrm{~B}$, these indicators suggest a worse prognosis for $\mathrm{CIMP}+$ patients than CIMP- patients in KIRC. This is consistent with our finding of recurrent $C D K N 2 A$ and $C D K N 2 B$ deletions in CIMP+ samples from KIRC patients mentioned earlier (Additional file 1: Figure S4), which were independently linked to a more clinically aggressive phenotype of kidney clear cell carcinoma [43].

In UCEC, we observed a strong association between CIMP status and histological subtype, wherein all the 68 samples of serous subtype had CIMP- labels and 103 of 161 endometrioid samples had CIMP+ labels, with 58 endometrioid samples being labeled as CIMP- (Additional file 1: Figure S6B, $P=9 \times 10^{-24}$, Fisher's exact test). These observations agree with our previous finding of a methylator phenotype occurring in endometrioid endometrial tumors but not serous endometrial tumors [25], as well as results reported by TCGA [26]. In fact, 
the association between CIMP status and histological subtype extends to tumor grade (an indicator of how quickly a tumor is likely to grow and spread based on microscopic appearance), where CIMP- samples exhibit higher grades than CIMP+ samples (Additional file 1: Figure S6B, $P=9.6 \times 10^{-4}$, Fisher's exact test, Bonferroni correction). In particular, all the high-grade samples were CIMP-, consistent with the fact that serous endometrial tumors tend to present higher grades than endometrioid endometrial tumors [46].

\section{Discussion}

Overall, our results support the existence of both commonalities and tissue-specific differences in CGI hypermethylation patterns across tumors. The most important similarity found in our analysis is the existence of consistent levels of average CGI hypermethylation that correlate with CIMP status and are independent of cancer type (Figure 2B,C). A consequence of this is the identification of a pan-cancer set of 89 genomic loci that can accurately separate CIMP+ from CIMP- samples across 12 different cancer types. Our genome-wide analyses (Additional file 1: Figure S1) show that much of the focal, cancer-related CGI hypermethylation occurs at loci that exhibit consistently baseline levels of methylation in control samples. This finding is particularly obvious and relevant for gene promoters (Additional file 1: Figure S7A). These data support a model whereby CIMP arises through mechanisms of de novo methylation that are largely reproducible events in the genome, rather than random spontaneous events. Furthermore, our data show unequivocally that this happens at a large number of genomic regions in a coordinated manner (Figure 1). Importantly, CGI methylation within gene bodies reveals that targets of hypermethylation in CIMP+ tumors are also found outside of promoters (Additional file 1 : Figures S7B,C).

Our results validate previous biological CIMP findings while unveiling potentially interesting new avenues of research. For example, significantly recurrent functional events in CIMP+ samples correspond to mutated genes or silencing of $M L H 1$ and MGMT, while recurrent events in CIMP - consist primarily of chromosomal amplifications and TP53 mutations (Table 2). This suggests a possible, previously unreported pan-cancer correspondence between the mutually exclusive $\mathrm{M}$ class and $\mathrm{C}$ class (groups dominated by mutations and copy number changes) as defined in Ciriello et al. [27] and the CIMP+ and CIMP- categories, respectively. Similarly, our analysis of upstream regulators that are shared across different sets of differentially methylated probes points to important members of the PRC such as EZH2 and SUZ12. This is consistent with previous reports of PRC targets being affected in CIMP tumors across cancers
[47], but our results involve a much larger collection of cancer types. While mutations in gene $H 3 F 3 A$, which encodes histone variant H3.3, have been correlated with specific DNA methylation subgroups in pediatric glioblastoma [19], our analysis of upstream regulators implicates involvement in most of the cancer types that we evaluated, with the exception of LUAD and BLCA (Figure 3B). The same upstream analysis suggests potential relevance in CIMP of several members of the sonic hedgehog pathway, which is consistent with tissue-specific patterns of aberrant CGI methylation. Loss-of-function mutations in the demethylating enzyme TET2 have been previously associated to CIMP in leukemia, and our results reveal recurrence of this mutation in CIMP+ for other types such as UCEC and READ (Figure 6A). Also, mutations of ARID1A have been linked to MSI and CIMP in gastrointestinal cancers, and our results indicate importance in UCEC and BLCA. BRAF mutations, which are perhaps one of the most commonly accepted indicator events for CIMP in colorectal cancers, also appear to be relevant in LUAD, but not the other tumor types.

Some of our results translate into biologically plausible hypotheses that could lead to refined treatment regimes. For example, amplification of genes PIK3CA and CCNE1 occur significantly more frequently in CIMP- samples. Interestingly, both PIK3CA and CCNE1 are directly or indirectly drug targetable [27], suggesting a possible combinational therapy aimed at CIMP- patients. Our pan-cancer regression tree revealed global CGI hypomethylation in samples with mutated NSD1, which came primarily from the HNSC data set (Additional file 1: Figure S2). The connection between NSD1 and DNA hypomethylation is likely related to a loss of its histone H3K36 methyltransferase activity that is a documented event in Sotos syndrome [48-51]. Our results also linked mutations in KDM6A, a H3K27me3 demethylase, to decreased $\mathrm{CpG}$ island methylation (Additional file 1: Figure S2). Notably, H3K27me3 recruits the polycomb repressive complex to specific targets, including HOX genes whose regulation is critical during cell-differentiation [52]. Furthermore, H3K27me3 has been proposed to 'pre-mark' genes for de novo methylation in cancer by favoring the aberrant recruitment of DNA methyltransferases [53], which suggests that KDM6A mutations may play an important role for the establishment of CIMP. Along the same lines, amplification of histone demethylase $K D M 5 A$, which targets H3K4me3/me2 active marks [54,55], was shown in our LUSC regression tree as exhibiting significant correlation with variations in average levels of CGI methylation (Additional file 1: Figure S4).

The results from our analysis of clinical annotations reveal ways in which our sample stratification can be used to refine current molecular subtyping. For example, 
an early study of DNA methylation patterns in breast cancer by Fang et al. had reported good survival for CIMP+ tumors [47]. However, more recent analyses have linked luminal B tumors, which are generally characterized by high levels of CGI methylation, with poor survival $[56,57]$. Our results help to clarify this apparent contradiction by showing that poor survival could be associated with luminal B patients with CIMP+ status and that luminal B patients with CIMP- can have good survival outcomes (Figure 8B). Interestingly, the situation is reversed in luminal A tumors, where CIMP+ status is associated to good survival and CIMP- status is associated with poor survival (as originally reported by Fang et al.). The lack of statistical significance for the reported differences might be due to the small number of available samples (for example, only five samples were available in the 'luminal B \& CIMP-' category), so the strong magnitude of effect that we observe should be re-evaluated in the context of a larger cohort. If confirmed, these results would suggest that the CIMP+/- and the luminal A/luminal $B$ categorizations can be combined to build improved prognosis indicators and also that current therapies are better suited for treating patients in the 'luminal B \& CIMP-' or 'luminal A \& CIMP+' categories than they are for treating patients in the 'luminal B \& CIMP+' or 'luminal A \& CIMP-' subgroups. This is a circumstance that needs to be further investigated.

\section{Conclusions}

Our tissue-specific selection of differentially methylated probes was important to identify concerted changes in average methylation levels that occur on top of the epigenetic background of tissue-specific repressive events. We have shown that those concerted changes are relevant for defining phenotypic and clinical differences among tumor samples. The cross-cancer analysis that we present also suggests that tissue-specific patterns may obscure detection of underlying pan-cancer epigenetic signals, which are often weak in comparison to competing signatures of cellular differentiation. Still, our results highlight the existence of several functional events that are relevant for CIMP across multiple cancer types and our set of 89 signature loci represents the first evidence for a pan-cancer methylation signature that can be used to classify multiple tumor types according to CIMP status.

We provide a robust, principled molecular stratification of solid tumors and cell lines based on CIMP signatures that can be reused in future studies to refine current molecular subtypes in a wide variety of cancers. By applying the same computational pipeline to samples from different tissues and cancer types, our work facilitates biologically meaningful cross-cancer comparisons. The many statistically significant associations between
CIMP status and both genomic and clinical features that we report in our work show that our CIMP+ and CIMPlabels define biologically distinct subpopulations whose phenotypic differences transcend DNA methylation patterns. Beyond several findings that characterize CIMP status in a tissue-specific manner, our study highlights the existence of important commonalities underlying CIMP as a pancancer epigenomic phenomenon. Still, our results are mostly correlational in nature and the identification of a unifying mechanism for CIMP across cancer types remains elusive. In order to further characterize causal genomic alterations that drive CIMP while answering the question of whether CIMP itself is a driver or a passenger trait for tumorigenesis and cancer progression, future pan-cancer studies shall benefit from extended experimental frameworks that include large scale interventions based on refined tumor stratification.

\section{Methods}

\section{Data}

\section{DNA methylation data from TCGA}

We downloaded level 3 data for 15 different cancer types from the TCGA data portal (https://tcga-data.nci.nih. gov/tcga/). Data had been acquired using the Illumina HumanMethylation450K platform and had been preprocessed following TCGA standard protocols. Data were downloaded in October 2013. The number of tumor and control samples that we downloaded for each cancer type is shown in Table 1. For the colorectal (COREAD) validation experiment, where we compared our sample classification algorithm with the methylation clusters defined by TCGA [24], we used a separate data set from the Illumina HumanMethylation27K platform. Specifically, we combined all the 320 samples from the COAD and READ cancer types for which both Illumina HumanMethylation27K methylation data and methylation cluster labels (CIMPH, CIMPL, Cluster3, Cluster4) were available.

Data pre-processing The data that we used had gone through all the pre-processing associated with level 3 data from TCGA. We discarded all the probes that interrogated locations in chromosomes $\mathrm{X}$ and $\mathrm{Y}$, as well as all probes that were masked as NA ('Not Available') for more than $90 \%$ of the samples. In the case of the KIRP dataset, we excluded nine tumor samples that behaved as outliers based on PCA plots computed over variably methylated probes (these tumor samples clustered together with each other, away from the rest of tumors and closer to the set of controls; the actual sample IDs were TCGA-A4-7915-01, TCGA-F9-A4JJ-01, TCGA-G76793-01, TCGA-GL-7966-01, TCGA-P4-A5E8-01, TCGAP4-A5EA-01, TCGA-BQ-5879-01, TCGA-BQ-5893-01, TCGA-BQ-5894-01). We normalized the data individually 
for every sample in every cancer type using the BMIQ method [58], which corrects for technical differences between type I probes and type II probes in the Illumina HumanMethylation platform. This method was chosen based on positive reviews from a recent study that compared several normalization methods [59]. A more detailed analysis of the technical biases associated with different Illumina probe types and their effect upon our probe selection criterion is provided in Additional file 2. We also provide an exploratory analysis of the impact of batch effects upon our sample stratification in Additional file 2.

\section{DNA methylation data from ENCODE}

We used DNA methylation data for 51 human cell lines from ENCODE (Additional file 5: Table S3). The data were downloaded from the HAIB Methyl450K track of the UCSC Human Genome Browser (https:// genome.ucsc.edu/) and had originally acquired using the Illumina HumanMethylation450K Bead Array platform, as was the case for the solid epithelial tumors data from TCGA. Data was pre-processed following the same guidelines that we had described for solid epithelial tumors.

\section{RNA-Seq data for TCGA samples}

Gene expression RNA-Seq data was downloaded from the TCGA data portal between January and June 2014 (Additional file 5: Table S5). We used RNA-Seq V2 data processed at level 3 . We used the files ending with 'rsem.genes.normalized_results' - these files contain gene expression values for 20,531 genes. Gene expression in these files is normalized so that the third quartile of genes with positive expression is set to 1000 , for each sample. We removed 29 genes that did not have a gene symbol. The COAD, READ, and UCEC data sets contained data from both the GA and HiSeq sequencing platforms. We merged these data as follows: if a given sample was present on both platforms, we kept only the HiSeq version. If only the GA or HiSeq version was present, then it was kept. Additional file 5: Table S5 contains the resulting number of samples in each cancer type.

\section{Selected functional event data for TCGA samples}

We downloaded data for 479 selected functional events across 3,299 TCGA samples that were made publicly available by the cBio group at Memorial Sloan Kettering Cancer Center (http://cbio.mskcc.org/cancergenomics/ pancan_tcga/), as described in Ciriello et al. [27]. We used the genomic alterations matrix file containing filtered calls only with date stamp of 5/31/2013.

\section{Clinical data for TCGA samples}

All the clinical data that we used in our analysis were downloaded from the UCSC Cancer Genomics Browser (https://genome-cancer.ucsc.edu/) [60]. All clinical data files had time stamp of $12 / 18 / 2013$. The actual set of available annotations varied across cancer types. Also, within each cancer type, the set of available annotations varied across samples.

\section{Statistical methods}

All our computations were done using the $\mathrm{R}$ statistical package (with the only exception of the $P$-values shown in Figure 3, which were computed directly using the IPA software). We used CpG island annotations from UCSC for hg19 and gene annotations provided by Illumina for their HumanMethylation450K platform.

\section{Statistical significance and biological relevance for probe selection thresholds}

Our approach to feature selection requires the use of two parameters that represent the maximum threshold for average methylation across controls $\left(\alpha_{C}\right)$ and the minimum threshold for average methylation across tumors $\left(\alpha_{\mathrm{T}}\right)$. A probe will be selected for inclusion into the differentially methylated set if and only if its average level of methylation computed over all the control samples is below $\alpha_{C}$ and its average level of methylation computed over all the tumor samples is above $\alpha_{\mathrm{T}}$. A choice of $\alpha_{C}=0.05$ and $\alpha_{T}=0.25$ seemed biologically reasonable to us in order to capture probes that exhibit consistently low levels of methylation in controls while presenting at least some sufficiently high level of methylation signal in tumors. We show statistical significance and biological relevance for this choice of thresholds:

(a) In order to evaluate statistical significance, we ran a random permutation experiment to estimate the number of false positive detections associated with this choice of parameters. More precisely, we considered each individual cancer type separately and we proceeded as follows: (1) we randomly shuffled the 'control' and 'tumor' labels for all the samples in the data set, (2) we applied our sample selection algorithm with parameters $\left(\alpha_{\mathrm{C}}=0.05, \alpha_{\mathrm{T}}=\right.$ 0.25 ) to the randomly shuffled data, and (3) we counted the number of selected features. These counts represent the number of features selected under the null hypothesis of randomly labeled samples (that is, when tumor $v$ s. control label assignment is independent of sample identifier), and therefore, they provide an estimate of the false positive rate associated to our feature selection procedure. The number of selected features averaged over 100 random permutations was below 0.1 for all types except READ (5.50) and STAD (502.32). The worst results were obtained for the cancer types with the lowest number of control samples, since this 
increases the probability of regions with low methylation being randomly aligned across those samples. When looking at our actual data, the number of selected features for READ was 1,255 , which leads to an acceptably low false detection rate. In the case of STAD, however, the rate between expected false detections and actual detections was around 50\% (502.32/1,110). Since only two controls were available for this cancer type at the time of our analysis, we found that any choice of threshold that guarantees a sufficiently low rate of false detections results in no differentially methylated probes being selected at all, so we decided to exclude STAD from most parts of our analysis.

(b) We evaluated biological relevance, in the sense of sufficiently large magnitude of effect. For this, we computed the average difference in mean per-probe methylation for samples in the CIMP+ vs. CIMPcategory (where labels had been learned using $\alpha_{C}=$ 0.05 and $\alpha_{\mathrm{T}}=0.25$ ) for each individual cancer type

(Additional file 1: Figure S9). For every cancer type, we observe differences in beta values of at least 0.1 and 0.3 when the variably methylated set and the differentially methylated set, respectively, are used to estimate average per-probe methylation in the CIMP+ and CIMP- subsets of samples. These mean differences are large enough to be considered biologically relevant (the TCGA marker paper on ovarian cancer [61], for example, proposes to use mean per-probe differences of 0.1 and 0.3 between tumors and controls as a relaxed and stringent threshold, respectively, in order to establish gene hypermethylation, while others such as Ciriello et al. rely on a single hard threshold of $0.1[27])$.

\section{Comparison to previously published hierarchical clustering results from TCGA}

For validation purposes and to address whether our classifications correspond convincingly to known examples of CIMP phenotypes from the literature, we compared our method with previously published results from TCGA that were also based on hierarchical clustering of DNA methylation levels. We applied our sample classification algorithm to an independent set of 320 colorectal samples (233 tumors and 87 controls) that had been previously analyzed by the TCGA Network [24] (see the 'Methods' section). These samples were used only for validation purposes and consisted of a mixture of 240 COAD samples (165 tumors and 75 controls) and 80 READ samples (68 tumors and 12 controls). Only a small subset of these samples overlapped our pan-cancer analysis (that is, 4 tumors and 38 controls were present in COAD and 1 tumor and 7 controls were present in READ from the pan-cancer data sets). Nevertheless, the data for these 320 TGCA samples derived from the HumanMethylation27K platform in contrast to the HumanMethylation450K data depicted in Table 1, enforcing that no measurements were reused in the two separate analyses. TCGA classified these samples into four different clusters (CIMPH, CIMPL, Cluster3, Cluster4) based on their overall levels of DNA methylation. Clusters $\mathrm{CIMPH}$ and CIMPL were described as having higher rates of methylation than the other two clusters. Our algorithm identified 86 samples as CIMP+, which all belonged to the CIMPH or CIMPL clusters (Additional file 1: Figure S10). Additionally, we identified 59 samples as CIMP-, which all belonged to the Cluster3 or Cluster4 categories. The remaining 88 tumors classified as CIMPi. The contingency table comparing the results from the two classification algorithms yielded a highly significant level of association based on Fisher's exact test $\left(P=2.15 \times 10^{-67}\right)$.

\section{Selection of pan-cancer differentially expressed genes in CIMP}

We evaluated individually each of the 3,892 CIMP + Hyper and 54 CIMP + Hypo regions that were associated to at least one known gene to search for significant correlations between DNA methylation and gene expression. In fact, since some of these regions were associated to more than one gene, we evaluated a total of 4,840 gene-region pairs. For each pair consisting of a gene and a CIMP $+{ }^{\text {Hyper }} /$ Hypo region, we computed the Spearman correlation between the average level of methylation measured by each individual probe in the region and the RNA-Seq level of expression measured for the gene. We then selected the probe with the highest absolute coefficient of correlation as the cluster representative. We did this separately for each of the 12 cancer types in our analysis. Figure $5 \mathrm{~B}$ shows the set of 121 genomic regions and 93 genes that exhibited significant levels of correlation $(\mathrm{FDR}<0.10)$ for all 12 cancer types.

\section{Analysis of selected functional events from Ciriello et al.}

Our analysis of differential frequencies in CIMP $+v s$. CIMP- samples was done by counting the number of samples in each of the two CIMP categories that presented each SFE for each individual type of cancer. Those counts were normalized by the total number of CIMP+ and CIMP- samples in each cancer type in order to turn them into frequencies of occurrence. For each SFE and each cancer type, we subtracted the frequency of occurrence in CIMP- samples from the frequency of occurrence in CIMP+ samples and we applied hierarchical clustering to draw the heat map shown in Figure 6A. We then pooled together all samples across different cancer types, and we computed global pancancer counts of occurrence for each selected functional event within the CIMP+ and the CIMP-subpopulations. 
We performed Fisher's exact test to evaluate associations between CIMP labels and sample counts for each individual SFE. We ranked the samples in terms of increasing $P$-values and we showed the top 20 scorers in Table 2 (including FDR values to correct for multiple hypothesis testing). For the comparison of the average number of mutation, amplification and deletion events per sample shown in Figure 6B, we provide a bar plot showing mean number of events of each category for each individual cancer type. Error bars show 95\% confidence intervals centered at the estimated means. The $P$ values shown in the figure correspond to a one-sided $t$-test. Finally, our analysis using binary decision trees was done with the R package 'partykit' [62], which provides tools for working with tree models for classification and regression. In the classification case, we restricted the analysis to samples that had been previously labeled as CIMP+ or CIMPand we used the CIMP status as class label. In the regression case, we worked with all the samples for which both methylation and SFEs data were available, and in the case of the pan-cancer tree (Additional file 1: Figure S2), we used the average level of methylation computed across all the probes in the pan-cancer union of variably methylated probe sets (for all the nine cancer types under consideration) as the response or dependent variable. Regression trees for individual cancer types (Additional file 1: Figure S4) were learned using the average level of methylation computed across all the probes in the cancer-specific variably methylated probe set as the response variable.

\section{Analysis of clinical annotations}

We divided our analysis of clinical annotations into two separate parts. First, we evaluated statistical associations between CIMP status and a number of clinical annotations that we considered inherently relevant to our study. This included age, gender, microsatellite instability, and overall survival. We evaluated associations between CIMP status and patient age at the time of diagnostic using the Kruskal-Wallis test for analysis of variance. We tested for statistical associations between CIMP labels and categorical clinical annotations using Fisher's exact test. In all these cases, we used Holm's correction for multiple hypotheses restricted to the number of cancer types tested for each individual annotation. We compared survival curves for CIMP+ vs. CIMP- tumors using the log-rank test (the $P$-values that we report for survival curve comparisons were not corrected for multiple hypotheses). For the second part of our study of clinical annotations, we did an exploratory analysis where we evaluated a set of 300 categorical clinical annotations, most of which were available only for a small subset of the 12 cancer types. In particular, we ran a total of 653 individual tests involving specific pairs of cancer type and annotation. Due to the exploratory nature of this part of our analysis, we applied the more conservative Bonferroni correction with a factor of 653 to correct for multiple hypothesis testing.

\section{Additional files}

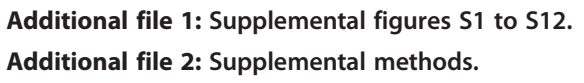

Additional file 4 CIMP labels for TCGA samples.

Additional file 5: Supplemental tables S1 to S7.

\section{Abbreviations}

BLCA: bladder urothelial carcinoma; BRCA: breast invasive carcinoma; CGI: CPG island; CIMP-: CIMP negative; CIMP: CPG island methylator phenotype; CIMP+: CIMP positive; CIMPi: CIMP intermediate; COAD: colon adenocarcinoma; EMT: epithelial-mesenchymal transition;

ENCODE: Encyclopedia of DNA Elements; HNSC: head and neck squamous cell carcinoma; IPA: Ingenuity Pathway Analysis; KIRC: kidney renal clear cell carcinoma; KIRP: kidney renal papillary cell carcinoma; LIHC: liver hepatocellular carcinoma; LOTO: leave-one-type-out; LUAD: lung adenocarcinoma; LUSC: lung squamous cell carcinoma; MSI: microsatellite instability; PAAD: pancreatic adenocarcinoma; PCA: principal component analysis; PRAD: prostate adenocarcinoma; PRC: polycomb repressor complex; READ: rectum adenocarcinoma; SD: standard deviation; SFE: selected functional event; STAD: stomach adenocarcinoma; TCGA: The Cancer Genome Atlas; THCA: thyroid carcinoma; UCEC: uterine corpus endometrioid carcinoma.

\section{Competing interests}

The authors declare that they have no competing interests.

\section{Authors' contributions}

FSV designed the study, performed the statistical analyses, and drafted the manuscript. He also downloaded and processed DNA methylation data for ENCODE cell lines, as well as clinical and functional data for TCGA tumors. VG downloaded and processed DNA methylation data from TCGA. GM downloaded and processed gene expression data from TCGA. VG and GM helped with data analysis, interpretation of results, and critical revision of the manuscript. LE conceived of the study and coordinated its design and technical implementation, while also helping to edit the manuscript. All authors read and approved the final manuscript.

\section{Acknowledgement}

This work was supported by the Intramural Program of the National Human Genome Research Institute, National Institutes of Health.

Received: 25 March 2015 Accepted: 30 March 2015

Published online: 17 April 2015

\section{References}

1. Robertson KD, Wolffe AP. DNA methylation in health and disease. Nat Rev Genet. 2000;1:11-9.

2. Jaenisch R, Bird A. Epigenetic regulation of gene expression: how the genome integrates intrinsic and environmental signals. Nat Genet. 2003;33(Suppl):245-54.

3. Portela A, Esteller M. Epigenetic modifications and human disease. Nat Biotechnol. 2010;28:1057-68.

4. Baylin SB. Aberrant patterns of DNA methylation, chromatin formation and gene expression in cancer. Hum Mol Genet. 2001;10:687-92.

5. Baylin SB, Jones PA. A decade of exploring the cancer epigenome - biological and translational implications. Nat Rev Cancer. 2011;11:726-34.

6. Rodríguez-Paredes M, Esteller M. Cancer epigenetics reaches mainstream oncology. Nat Med. 2011;17:330-9.

7. Esteller M, Herman JG. Cancer as an epigenetic disease: DNA methylation and chromatin alterations in human tumours. J Pathol. 2002;196:1-7. 
8. Issa J-PJ. DNA methylation as a therapeutic target in cancer. Clin Cancer Res. 2007;13:1634-7.

9. Esteller M. Cancer epigenetics for the 21st century: what's next? Genes Cancer. 2011:2:604-6.

10. Egger $G$, Liang G, Aparicio A, Jones PA. Epigenetics in human disease and prospects for epigenetic therapy. Nature. 2004;429:457-63.

11. Hughes LAE, Melotte V, de Schrijver J, de Maat M, Smit VTHBM, Bovée JVMG, et al. The CpG island methylator phenotype: what's in a name? Cancer Res. 2013;73:5858-68.

12. Issa J-P. CpG island methylator phenotype in cancer. Nat Rev Cancer. 2004:4:988-93.

13. Toyota M, Ahuja N, Ohe-Toyota M, Herman JG, Baylin SB, Issa J-PJ. CpG island methylator phenotype in colorectal cancer. Proc Natl Acad Sci. 1999:96:8681-6.

14. Hughes LAE, Khalid-de Bakker CAJ, Smits KM, van den Brandt PA, Jonkers D, Ahuja $\mathrm{N}$, et al. The CpG island methylator phenotype in colorectal cancer: progress and problems. Biochim Biophys Acta. 1825;2012:77-85.

15. Arain MA, Sawhney M, Sheikh S, Anway R, Thyagarajan B, Bond JH, et al. CIMP status of interval colon cancers: another piece to the puzzle. Am J Gastroenterol. 2010;105:1189-95.

16. Grady WM. CIMP and colon cancer gets more complicated. Gut. 2007;56:1498-500.

17. Curtin K, Slattery ML, Samowitz WS. CpG island methylation in colorectal cancer: past, present and future. Patholog Res Int. 2011;2011:902674.

18. Weisenberger DJ. Characterizing DNA, methylation alterations from The Cancer Genome Atlas. J Clin Invest. 2014;124:17-23.

19. Witte T, Plass C, Gerhauser C. Pan-cancer patterns of DNA methylation. Genome Med. 2014;6:66

20. Shen H, Laird PW. Interplay between the cancer genome and epigenome. Cell. 2013;153:38-55.

21. Kim JH, Karnovsky A, Mahavisno V, Weymouth T, Pande M, Dolinoy DC, et al. LRpath analysis reveals common pathways dysregulated via DNA methylation across cancer types. BMC Genomics. 2012;13:526.

22. Sproul D, Kitchen RR, Nestor CE, Dixon JM, Sims AH, Harrison DJ, et al. Tissue of origin determines cancer-associated $\mathrm{CpG}$ island promoter hypermethylation patterns. Genome Biol. 2012;13:R84.

23. Kalari S, Pfeifer GP. Identification of driver and passenger DNA methylation in cancer by epigenomic analysis. Adv Genet. 2010;70:277-308.

24. Research TCGA. Network. Comprehensive molecular characterization of human colon and rectal cancer. Nature. 2012;487:330-7.

25. Kolbe DL, DeLoia JA, Porter-Gill P, Strange M, Petrykowska HM, Guirguis A, et al. Differential analysis of ovarian and endometrial cancers identifies a methylator phenotype. PLoS One. 2012;7:e32941.

26. Research TCGA. Network. Integrated genomic characterization of endometrial carcinoma. Nature. 2013:497:67-73.

27. Ciriello G, Miller ML, Aksoy BA, Senbabaoglu Y, Schultz N, Sander C. Emerging landscape of oncogenic signatures across human cancers. Nat Genet. 2013;45:1127-33.

28. Sánchez-Vega F, Gotea V, Petrykowska HM, Margolin G, Krivak TC, DeLoia JA, et al. Recurrent patterns of DNA methylation in the ZNF154, CASP8, andVHL promoters across a wide spectrum of human solid epithelial tumors and cancer cell lines. Epigenetics. 2013;8:1355-72.

29. Hansen KD, Timp W, Bravo HC, Sabunciyan S, Langmead B, McDonald OG, et al. Increased methylation variation in epigenetic domains across cancer types. Nat Genet. 2011:43:768-75.

30. Fernando RI, Litzinger M, Trono P, Hamilton DH, Schlom J, Palena C. The T-box transcription factor Brachyury promotes epithelial-mesenchymal transition in human tumor cells. J Clin Invest. 2010;120:533-44.

31. Messner S, Hottiger MO. Histone ADP-ribosylation in DNA repair, replication and transcription. Trends Cell Biol. 2011;21:534-42.

32. Kato J, Zhu J, Liu C, Stylianou M, Hoffmann V, Lizak MJ, et al. ADP-ribosylarginine hydrolase regulates cell proliferation and tumorigenesis. Cancer Res. 2011;71:5327-35.

33. Shain AH, Pollack JR. The spectrum of SWI/SNF mutations, ubiquitous in human cancers. PLoS One. 2013;8:e55119.

34. Butler JS, Dent SYR. Chromatin "resetting" during transcription elongation: a central role for methylated H3K36. Nat Struct Mol Biol. 2012;19:863-4.

35. Song MS, Salmena L, Pandolfi PP. The functions and regulation of the PTEN tumour suppressor. Nat Rev Mol Cell Biol. 2012;13:283-96.

36. Roberts PJ, Der CJ. Targeting the Raf-MEK-ERK mitogen-activated protein kinase cascade for the treatment of cancer. Oncogene. 2007;26:3291-310.
37. Weisenberger DJ, Siegmund KD, Campan M, Young J, Long TI, Faasse MA, et al. CpG island methylator phenotype underlies sporadic microsatellite instability and is tightly associated with BRAF mutation in colorectal cancer. Nat Genet. 2006:38:787-93.

38. Ogino S, Kawasaki T, Kirkner GJ, Loda M, Fuchs CS. CpG island methylator phenotype-low (CIMP-low) in colorectal cancer: possible associations with male sex and KRAS mutations. J Mol Diagn. 2006;8:582-8.

39. Serra RW, Fang M, Park SM, Hutchinson L, Green MR. A KRAS-directed transcriptional silencing pathway that mediates the CpG island methylator phenotype. Elife. 2014;3:e02313.

40. De Vogel S, Weijenberg MP, Herman JG, Wouters KAD, de Goeij AFPM, van den Brandt PA, et al. MGMT and MLH1 promoter methylation versus APC, KRAS and BRAF gene mutations in colorectal cancer: indications for distinct pathways and sequence of events. Ann Oncol. 2009;20:1216-22.

41. The Cancer Genome Atlas Research Network. Comprehensive molecular characterization of urothelial bladder carcinoma. Nature. 2014;507:315-22.

42. Research TCGA. Network. Comprehensive molecular portraits of human breast tumours. Nature. 2012;490:61-70.

43. La Rochelle J, Klatte T, Dastane A, Rao N, Seligson D, Said J, et al. Chromosome 9p deletions identify an aggressive phenotype of clear cell renal cell carcinoma. Cancer. 2010;116:4696-702.

44. Yamauchi M, Morikawa T, Kuchiba A, Imamura Y, Qian ZR, Nishihara R, et al. Assessment of colorectal cancer molecular features along bowel subsites challenges the conception of distinct dichotomy of proximal versus distal colorectum. Gut. 2012;61:847-54.

45. Soreide K. High-fidelity of five quasimonomorphic mononucleotide repeats to high-frequency microsatellite instability distribution in early-stage adenocarcinoma of the colon. Anticancer Res. 2011:31:967-71.

46. Nakayama K, Nakayama N, Ishikawa M, Miyazaki K. Endometrial serous carcinoma: its molecular characteristics and histology-specific treatment strategies. Cancers (Basel). 2012;4:799-807.

47. Fang F, Turcan S, Rimner A, Kaufman A, Giri D, Morris LGT, et al. Breast cancer methylomes establish an epigenomic foundation for metastasis. Sci Transl Med. 2011;3:75ra25.

48. Faravelli F. NSD1 mutations in Sotos syndrome. Am J Med Genet C Semin Med Genet. 2005;137C:24-31

49. NSD1 mutations in Sotos syndrome alter the DNA methylation landscape of genes involved in somatic growth and neuronal transmission. [http:// www.ashg.org/2012meeting/abstracts/fulltext/f120122152.htm]

50. A specific DNA methylation signature associated with NSD1+/- mutations in Sotos syndrome reveals a significant genome-wide loss of DNA methylation (DNAm) targeting CGs in regulatory regions of key developmental genes. [http://www.ashg.org/2013meeting/abstracts/fulltext/f130121963.htm].

51. Wagner EJ, Carpenter PB. Understanding the language of Lys 36 methylation at histone H3. Nat Rev Mol Cell Biol. 2012;13:115-26.

52. Agger $\mathrm{K}$, Cloos PAC, Christensen J, Pasini D, Rose S, Rappsilber J, et al. UTX and JMJD3 are histone H3K27 demethylases involved in HOX gene regulation and development. Nature. 2007;449:731-4.

53. Schlesinger Y, Straussman R, Keshet I, Farkash S, Hecht M, Zimmerman J, et al. Polycomb-mediated methylation on Lys 27 of histone H3 pre-marks genes for de novo methylation in cancer. Nat Genet. 2007;39:232-6.

54. Hou J, Wu J, Dombkowski A, Zhang K, Holowatyj A, Boerner JL, et al. Genomic amplification and a role in drug-resistance for the KDM5A histone demethylase in breast cancer. Am J Transl Res. 2012;4:247-56.

55. Rasmussen PB, Staller P. The KDM5 family of histone demethylases as targets in oncology drug discovery. Epigenomics. 2014;6:277-86.

56. Stefansson OA, Moran S, Gomez A, Sayols S, Arribas-Jorba C, Sandoval J, et al. A DNA methylation-based definition of biologically distinct breast cancer subtypes. Mol Oncol. 2014;9:555-68.

57. Conway K, Edmiston SN, May R, Kuan P, Chu H, Bryant C, et al. DNA methylation profiling in the Carolina Breast Cancer Study defines cancer subclasses differing in clinicopathologic characteristics and survival. Breast Cancer Res. 2014;16:450.

58. Teschendorff AE, Marabita F, Lechner M, Bartlett T, Tegner J, Gomez-Cabrero $D$, et al. A beta-mixture quantile normalization method for correcting probe design bias in Illumina Infinium 450 k DNA methylation data. Bioinformatics. 2013:29:189-96.

59. Marabita F, Almgren M, Lindholm ME, Ruhrmann S, Fagerström-Billai F, Jagodic M, et al. An evaluation of analysis pipelines for DNA methylation profiling using the Illumina HumanMethylation450 BeadChip platform. Epigenetics. 2013;8:333-46. 
60. Cline MS, Craft B, Swatloski T, Goldman M, Ma S, Haussler D, et al. Exploring TCGA pan-cancer data at the UCSC Cancer Genomics Browser. Sci Rep. 2013:3:2652.

61. Research TCGA. Network. Integrated genomic analyses of ovarian carcinoma. Nature. 2011;474:609-15.

62. Hothorn, Torsten and Zeileis, Achim, (2014), partykit: A Modular Toolkit for Recursive Partytioning in R, Working Papers, Faculty of Economics and Statistics, University of Innsbruck, http://EconPapers.repec.org/

RePEc:inn:wpaper:2014-10.

Submit your next manuscript to BioMed Central and take full advantage of:

- Convenient online submission

- Thorough peer review

- No space constraints or color figure charges

- Immediate publication on acceptance

- Inclusion in PubMed, CAS, Scopus and Google Scholar

- Research which is freely available for redistribution 